\title{
Sorting in the Presence of Misperceptions
}

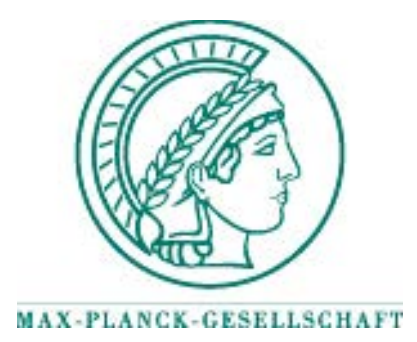

Max Planck Institute for

Tax Law and Public Finance

Department of Business and Tax Law Department of Public Economics

http:/ / www.tax.mpg.de 
Working papers of the Max Planck Institute for Tax Law and Public Finance Research Paper Series serve to disseminate the research results of work in progress prior to publication to encourage the exchange of ideas and academic debate. Inclusion of a paper in the Research Paper Series does not constitute publication and should not limit publication in any other venue. The preprints published by the Max Planck Institute for Tax Law and Public Finance represent the views of the respective author(s) and not of the Institute as a whole. Copyright remains with the author(s).

Max Planck Institute for Tax Law and Public Finance

Marstallplatz 1

D-80539 Munich

Tel: $\quad+498924246-0$

Fax: $\quad+498924246-501$

E-mail: ssrn@tax.mpg.de

http://www.tax.mpg.de 


\title{
Sorting in the Presence of Misperceptions*
}

\author{
Lisa Windsteiger ${ }^{\dagger}$
}

\begin{abstract}
In this paper I develop a general model of how social segregation and beliefs interact. Sorting decisions will be affected by beliefs about society, but these beliefs about society are in turn influenced by social interactions. In my model, people sort into social groups according to income, but become biased about the income distribution once they interact only with their own social circle. I define "biased sorting equilibria", which are stable partitions in which people want to stay in their chosen group, despite their acquired misperceptions about the other groups. I introduce a refinement criterion - the consistency requirement - and find necessary and sufficient conditions for existence and uniqueness of biased sorting equilibria.
\end{abstract}

JEL Classification: D83, D85, Z13

Keywords: Stratification, Assortative Matching, Group Formation, Beliefs

*I thank Ronny Razin, Matt Levy and the participants of the LSE Microeconomic Theory Work in Progress Seminar for their helpful suggestions and comments.

${ }^{\dagger}$ Max Planck Institute for Tax Law and Public Finance, Email: Lisa.Windsteiger@tax.mpg.de 


\section{Introduction}

Who we choose to socialize with is often determined by our beliefs about others, about their qualities and their characteristics, and by our surmise about what effects their good and bad traits will have on ourselves. It is natural that we try to interact frequently with people who we think we can benefit from, be it in a material sense or simply because we enjoy their company.

On the other hand, our beliefs about society are likely to be influenced by our social interactions, by what and who we observe on a day-to-day basis. Depending on how diverse our social circles are, we might end up knowing a lot or very little about certain groups in society. Specifically, if we do not interact with some social groups, we are prone to develop distorted beliefs about what people are like in that group, about their characteristics and their traits. This in turn might influence who we choose to interact with in the first place and hence solidify and reinforce our attitudes and beliefs.

Take for example the question of how contact with ethnic minorities affects people's attitudes towards minorities. Dustmann and Preston (2001) show that looking at the effect of living in an area with high ethnic diversity on attitudes towards minorities can give a misleading answer. The reason for this is that we can at least to some degree decide where we want to live, and therefore people might live in ethnically diverse areas because they have a favourable attitude towards minorities in the first place - there is a two-way interaction between location choice and people's beliefs that needs to be taken into account.

Another example is parents' school choice for their kids. There is considerable evidence (see e.g. a 2007 Center on Education Policy report using National Educational Longitudinal Study (NELS) data from 1988-2000) that private schools and state schools yield relatively similar learning outcomes if we control for pupils' family background. ${ }^{1}$ Nevertheless, parents are willing to pay a lot to live in areas with supposedly "good" schools (especially in the US) or to send their kids to private schools. However, these

\footnotetext{
${ }^{1}$ On a related note, Abdulkadiroglu et al. (2014) show that high achieving peers and racial composition of schools have no effect on learning achievement of individual pupils.
} 
seem to be mainly parents who were privately educated themselves. In fact, Evans and Tilley (2011) show that in the UK parents who went to private schools are five times more likely to send their kids to private schools than state-educated parents (controlling for income). Levy and Razin (2016) describe a model in which beliefs about the benefits of private education are passed on from parents to their children and influence school choice (and subsequent success in the labour market) from generation to generation.

If we want to examine how changes in the economy - like an increase in income inequality, a reform to the education system or a surge in immigration - affect social groups and the belief system in society, we have to bear in mind that it doesn't suffice to look at the direct effect that these changes have on segregation and beliefs. Where society will end up in the long run depends also on the mutual reinforcement and interdependence of social segregation and beliefs.

The interaction of social segregation and beliefs about society is what I examine in this paper. I take the canonical model of sorting according to income as a starting point. In this model, all feasible partitions of the income distribution are monotone, i.e. social groups will be single intervals of the income distribution. It is important to note, though, that without further assumptions on the sorting fees there is nothing that pins down the exact way in which society will segregate in this model, i.e. how the social groups will look like. By varying the menu of sorting fees accordingly, any monotone partition of the income distribution is feasible. Furthermore, while this model takes into account that our beliefs about society affect our social interactions, it doesn't allow for the effect to go in the other direction: people's beliefs about the whole of society remain unchanged (and unbiased), even if people interact mainly with their own social circle.

In the present paper, I eliminate these shortcomings by adding misperceptions to the model. I demonstrate that this addresses both issues of the canonical model at the same time. First, it accounts for the fact that our social interactions shape our beliefs about society, and second it limits the amount of partitions that are feasible in equilibrium and therefore reduces multiplicity.

In my model of sorting with misperceptions I assume that, once society is segregated and people interact mainly with their own social circle, they 
become biased about the overall income distribution, and specifically about average income in the other groups. I define as "biased sorting equilibria" those partitions of the income distribution that are stable given people's misperceptions, i.e. partitions in which people want to stay in their chosen group, despite their acquired distorted beliefs.

I show that adding misperceptions to the model initially leads to more complications: While in the canonical model all equilibrium partitions are monotone (i.e. single, connected intervals), biased sorting equilibria can also be non-monotone and hence people in one and the same group can have very different incomes, which complicates the analysis and is at odds with empirical evidence of assortative matching. Furthermore, the issue of multiplicity of equilibrium partitions persists, and even for a given equilibrium partition the sorting fees might not be uniquely determined. Finally, people's beliefs about other groups can be inconsistent with what they see: they can be surprised by seeing people with certain incomes choosing to be in certain groups, because given their beliefs about incomes in the other groups they do not think these choices are optimal.

In order to address these problems, I introduce a refinement criterion that I call the "consistency requirement". A partition satisfies consistency if the misperceptions are such that people are not surprised by the choices of people in other groups. I show that all biased sorting equilibria with consistency are monotone, and that the menu of sorting fees is uniquely pinned down for a given equilibrium partition. Furthermore, I demonstrate that if there are two groups in society and the misperceptions satisfy a form of monotonicity, then the consistency requirement selects a unique biased sorting equilibrium out of all possible stable partitions. In that case, social groups and sorting fees are uniquely determined. In the last section I examine under which conditions on the belief function biased sorting equilibria with consistency and more than two groups will exist.

The rest of this paper is organized as follows. Section 2 discusses related literature, Section 3 presents the canonical model of sorting with respect to income and Section 4 introduces misperceptions into that model and explains the concept of biased sorting equilibrium and the consistency requirement in its local and global form. Section 5 finds conditions on the functional form of the misperceptions that lead to existence and uniqueness 
of binary biased sorting equilibria with consistency. Section 6 examines under which conditions biased sorting equilibria with consistency and more than two groups can exist. Section 7 concludes.

\section{Relation to existing literature}

The canonical model of sorting and assortative matching was most famously employed by Becker (1974) to model the marriage market. Pesendorfer (1995) uses it to explain fashion cycles. Rayo (2013) examines optimal sorting from a profit-maximizing monopolist's point of view, Damiano and $\mathrm{Li}$ (2007) analyze the case of two or more competing firms and optimality with respect to welfare is explored in Levy and Razin (2015). In Windsteiger (2017c), I compare the optimal partitions for the monopolist and the social planner and find that the optimal type of sorting depends on the shape of the income distribution and varies depending on which entity (profit-maximizing monopolist or benevolent social planner) is assumed to offer the sorting technology.

What all the above papers have in common is that beliefs about society determine who people interact with, but social interactions do not influence beliefs. In fact, people retain perfect knowledge about society despite interacting only with a (potentially small) group of society.

Recently, the fact that segregation can affect beliefs has gained attention in the literature: On the theoretical side, Golub and Jackson (2012) present a model in which homophily (and resulting segregation) slows down convergence to a consensus in society. Concerning empirical evidence, Algan et al. (2015) show that political views converge among peers at university, and Boisjoly et al. (2006) and Burns et al. (2013) find that having roommates of a different ethnicity to one's own lowers students' prejudices.

That (potentially biased) beliefs can, in turn, have an effect on segregation, is pointed out by Dustmann and Preston (2001). They argue that estimating the effect of living in ethnically diverse neighbourhoods on attitudes towards minorities can lead to biased results, if we do not take into account how those attitudes affect neighbourhood choices in the first place. Levy and Razin (2016) present a model in which beliefs about school quality and parent's school choice for their children interact to create essentially 
two groups of society: a group of privately educated parents who believe in the benefits of the private school system and send their children to private school as well, and a group of state educated parents, who think private schools are not worth paying for and send their kids to state schools.

The main contribution of my paper is to present a general model in which beliefs about society and segregation choice interact to create an endogenous system of beliefs and societal groups. This general model can be used to analyze sorting according to many variables that are distributed continuously in society. ${ }^{2}$ While in my version of the model, I assume that people sort according to income, the continuous variable could also be "ability" or "intelligence" and the model could be about sorting into different types of schools.

\section{A theoretical model of economic segrega- tion}

Let income $y$ in an economy be distributed according to some income distribution $F(y)$ on the interval $Y=\left[0, y_{\max }\right]$ where $y_{\max }<\infty$. Assume furthermore that $F(y)$ is continuous and strictly monotonic.

Suppose that an agent's utility is increasing not only in her own income but also in the average income of the people that she interacts with, which I will henceforth call her "reference group". Specifically, I will assume that a person with income $y_{j}$ gets utility $U_{j}=U\left(y_{j}, E\left(y \mid y \in S_{i}\right)\right)$, where $S_{i}$ is individual $j$ 's reference group. If there is no economic segregation, let everybody's reference group be a representative sample of the whole population, such that $U_{j}=U\left(y_{j}, E(y)\right)$. However, suppose that a person with income $y_{j}$ can pay $b_{i}(i \in\{1, . ., n\})$ to join club $S_{b_{i}}(i \in\{1, . ., n\})$ and get utility

$$
U\left(y_{j}, E\left[y \mid y \in S_{b_{i}}\right]\right)-b_{i}
$$

\footnotetext{
${ }^{2}$ Due to strict increasingness of $U$ those should, however, be variables where the whole of society agrees that "more is better", such as intelligence, ability, income or wealth.
} 
or refrain from paying any $b_{i}$ and get

$$
U\left(y_{j}, E\left[y \mid y \in S_{b_{0}}\right]\right)
$$

where $S_{b_{i}}$ is the set of incomes $y$ of people who have paid $b_{i}$ and $b_{0}=0$. Let $U(.,$.$) be continuous, strictly increasing in both arguments and strictly$ supermodular, such that ${ }^{3}$

$$
\forall x^{\prime}>x: U\left(y, x^{\prime}\right)-U(y, x) \text { is strictly increasing in } y \text {. }
$$

Then I can define the following:

Definition 1 A sorting equilibrium is a partition $\left[S_{b_{0}}, S_{b_{1}}, . ., S_{b_{n}}\right]$ of $Y$ and a menu of sorting fees $\left[b_{0}, b_{1}, . ., b_{n}\right]$ (with $b_{i}<b_{i+1} \forall i$ and $\left.b_{0}=0\right)$ such that $\forall i \in\{0, . ., n\}$

$$
\begin{aligned}
& U\left(y, E\left[y \mid y \in S_{b_{i}}\right]\right)-b_{i} \geq U\left(y, E\left[y \mid y \in S_{b_{k}}\right]\right)-b_{k} \quad \forall y \in S_{b_{i}}, \forall i, \forall k \neq i \\
\Leftrightarrow & U\left(y, E\left[y \mid y \in S_{b_{i}}\right]\right)-U\left(y, E\left[y \mid y \in S_{b_{k}}\right]\right) \geq b_{i}-b_{k} \quad \forall y \in S_{b_{i}}, \forall i, \forall k \neq i
\end{aligned}
$$

In a sorting equilibrium as defined above people stay in the group that gives them the highest utility.

Corollary 1 In any sorting equilibrium, groups with a higher average income correspond to higher sorting fees.

Proof. This immediately follows from Definition 1, from the assumption that all $b_{i}$ are different and from the fact that $U$ is strictly increasing in both arguments.

I can show that all sorting equilibria will be of a certain form:

\footnotetext{
${ }^{3}$ Note that this paper offers only a very reduced form model of economic segregation. That people's utility is increasing in the average income of the other people they mix with is perhaps a simplified way of saying that living in an affluent neighborhood offers many benefits, such as good schools (because people are willing to spend more on the education of their kids, and because the presence of children of rich people might increase other pupil's chances in life through various peer effects) and pleasant surroundings such as parks or leisure centres (perhaps with increased security or surveillance). Instead of modelling all this on a micro level, I subsum all these effects into a utility function which is increasing in the average income of one's peers.
} 
Proposition 1 All sorting equilibria will be monotone. ${ }^{4} 5$

Proof. Suppose w.l.o.g. that a sorting equilibrium exists where $y_{2} \in S_{b_{i}}$ and $y_{1} \in S_{b_{j}}$, with $b_{i}<b_{j}$ but $y_{2}>y_{1}$. Then I must have

$$
U\left(y_{2}, E\left[y \mid y \in S_{b_{j}}\right]\right)-U\left(y_{2}, E\left[y \mid y \in S_{b_{i}}\right]\right) \leq b_{j}-b_{i}
$$

and

$$
U\left(y_{1}, E\left[y \mid y \in S_{b_{j}}\right]\right)-U\left(y_{1}, E\left[y \mid y \in S_{b_{i}}\right]\right) \geq b_{j}-b_{i}
$$

and hence

$U\left(y_{1}, E\left[y \mid y \in S_{b_{j}}\right]\right)-U\left(y_{1}, E\left[y \mid y \in S_{b_{i}}\right]\right) \geq U\left(y_{2}, E\left[y \mid y \in S_{b_{j}}\right]\right)-U\left(y_{2}, E\left[y \mid y \in S_{b_{i}}\right]\right)$.

But due to $y_{2}>y_{1}$ this is a contradiction to $U$ being strictly supermodular.

Proposition 1 allows me to rewrite the definition of a sorting equilibrium in terms of intervals of $Y$.

Corollary 2 A sorting equilibrium is characterized by a partition $\left[0, \hat{y}_{1}, \ldots, \hat{y}_{n}, y_{\max }\right]$ of $Y$ and a menu of sorting fees $\left[b_{0}, b_{1}, . ., b_{n}\right]\left(\right.$ with $b_{i}<b_{i+1} \forall i$ and $\left.b_{0}=0\right)$ such that $\forall i \in\{1, . ., n\}$

$$
\begin{aligned}
& U\left(\hat{y}_{i}, E\left[y \mid y \in S_{b_{i}}\right]\right)-b_{i}=U\left(\hat{y}_{i}, E\left[y \mid y \in S_{b_{i-1}}\right]\right)-b_{i-1} \quad \forall i \\
\Leftrightarrow & U\left(\hat{y}_{i}, E\left[y \mid y \in S_{b_{i}}\right]\right)-U\left(\hat{y}_{i}, E\left[y \mid y \in S_{b_{i-1}}\right]\right)=b_{i}-b_{i-1} \quad \forall i
\end{aligned}
$$

Proof. Given the fact that $S_{b_{i}}=\left[\hat{y}_{i}, \hat{y}_{i+1}\right] \forall i$ and equilibrium condition (1), it follows that both

$$
U\left(y, E\left[y \mid y \in S_{b_{i}}\right]\right)-U\left(y, E\left[y \mid y \in S_{b_{i-1}}\right]\right) \geq b_{i}-b_{i-1} \quad \forall y \in\left[\hat{y}_{i}, \hat{y}_{i+1}\right], \forall i
$$

\footnotetext{
${ }^{4}$ By monotone I mean that the groups $S_{b_{i}}$ are single intervals of $Y$. (By Corollary 1, this implies that those groups sitting higher up on the $Y$ scale correspond to higher sorting fees.)

${ }^{5}$ If some or all $b_{i}$ are equal, then there exist trivial non-monotone sorting equilibria where the average income in all those groups with the same $b_{i}$ is the same, so that people are indifferent about which of these groups to join. I exclude those cases from my analysis by assuming that $b_{i}<b_{i+1}$.
} 
and

$$
U\left(y, E\left[y \mid y \in S_{b_{i}}\right]\right)-U\left(y, E\left[y \mid y \in S_{b_{i-1}}\right]\right) \leq b_{i}-b_{i-1} \quad \forall y \in\left[\hat{y}_{i-1}, \hat{y}_{i}\right], \forall i
$$

need to hold in any sorting equilibrium. This implies that a person with income $\hat{y}_{i}$ just at the border of two groups $S_{b_{i-1}}$ and $S_{b_{i}}$ has to be exactly indifferent between joining either of the two groups in equilibrium. Hence, we get

$$
U\left(\hat{y}_{i}, E\left[y \mid y \in S_{b_{i}}\right]\right)-U\left(\hat{y}_{i}, E\left[y \mid y \in S_{b_{i-1}}\right]\right)=b_{i}-b_{i-1} \quad \forall i
$$

Corollary 3 For a given equilibrium partition $\left[0, \hat{y}_{1}, \ldots, \hat{y}_{n}, y_{\max }\right]$, the menu of sorting fees $\left[b_{0}, b_{1}, . ., b_{n}\right]$ (with $b_{i}<b_{i+1} \forall i$ and $b_{0}=0$ ) is always unique.

Proof. This follows immediately from equilibrium condition (2).

The above presented canonical model of sorting according to income has many positive features: The equilibrium partitions will always be monotone and therefore individual income within the resulting equilibrium groups will be similar (or at least within a simple interval), which simplifies the analysis of the model and is also compatible with empirical evidence of segregation according to income and assortative matching. Furthermore, inherent in the above definition is a notion of consistency: if a person sees the income of another person and knows which group this person joined, she always thinks that this person is correct in doing so, because both people evaluate the benefit of being in a certain group (given a certain income) equally. This means that no extra condition is needed to guarantee consistency, it "comes for free" in the equilibrium condition (2) - if a person in a certain group thinks that being in that group is best for her, then also all other people - no matter which group they belong to - will think that this is optimal for her.

However, from equilibrium condition (2) it is immediate to see that the model delivers no prediction about the type of segregation that will happen in a society, i.e. how the social groups will look like: For any continuous distribution function $F$ there exists an infinite number of equilibrium partitions. More specifically, for any partition $P=\left[0, \hat{y}_{1}, \ldots, \hat{y}_{n}, y_{\max }\right]$ there 
exists a menu of sorting fees $\mathbf{b}=\left[0, b_{1}, \ldots b_{n}\right]$ such that $(P, \mathbf{b})$ is a sorting equilibrium. Moreover, while in this model people's beliefs about society determine their social interactions, the reverse effect is not taken into account: segregation has no effect on people's beliefs about the economy and people retain perfect knowledge about the income distribution in the whole of society, even though they interact mainly with a select group of people who are similar to them in terms of income.

One way to try and resolve the issue of multiplicity is to look at the supply side of the sorting technology: we can analyze the optimal partition that a profit-maximizing monopolist, a number of competing firms or a benevolent social planner would want to offer and thereby select "plausible" equilibria out of the infinite number of possible equilibria. I explore this path in another paper of mine and find that the form of resulting optimal partitions depends on the underlying distribution function and on which entity is assumed to provide the sorting technology (see Windsteiger $(2017 \mathrm{c}))$.

In this paper, I pursue a different path: I add misperceptions to the model. Specifically, I will assume that people, once they are sorted into their respective groups, become biased about average income in the other groups, and I will define partitions as biased sorting equilibria if they are such that people want to stay in their group given their misperceptions about the rest of society. I will show in the next section that this addresses both of the above mentioned limitations of the canonical model: First, it lifts the assumption that people retain perfect knowledge about society once they are sorted and therefore allows for the interaction between segregation and beliefs to go both ways. Second, restricting attention to biased sorting equilibria with the additional requirement of consistency (which I will explain below) greatly reduces the number of possible equilibrium partitions and can lead, if the misperceptions are of a certain form, even to uniqueness.

\section{Sorting with misperceptions}

Suppose that people, once they are sorted into their group $S_{b_{i}}$, become biased about average income in the other groups and hence about the 
overall income distribution. I will model a group's belief about the other groups as resulting from a group belief "technology". Specifically, I will assume that for any partition of $Y$ with $n+1$ groups, people's biased perception of the average income of the other groups can be characterized by the belief function

$$
B: \mathbf{P} \rightarrow Y^{(n+1)^{2}}
$$

(where $\mathbf{P}$ is the space of all partitions $P=\left[S_{0}, S_{b_{1}}, . . S_{b_{n}}\right]$ of $Y$ ) that maps every partition of $Y$ into an $(n+1)^{2}$-dimensional vector of beliefs

$$
\left(E_{0}^{0}(P), E_{0}^{1}(P), \ldots E_{0}^{n}(P), E_{1}^{0}(P), E_{1}^{1}(P), \ldots, E_{n}^{n-1}(P), E_{n}^{n}(P)\right)
$$

where $E_{i}^{j}(P)$ denotes group $i$ 's belief about average income in group $j$ and hence the first $n+1$ entries of (3) denote group $S_{b_{0}}$ 's belief about average income in $S_{b_{0}}$ and all the other groups, entry $n+2$ to $2(n+1)$ denote group $S_{b_{1}}$ 's belief about average income in all groups, etc.... I will assume that the belief function is such that people are correct about average income in their own group, i.e.

$$
E_{i}^{i}(P)=E^{i}(P) \quad \forall i
$$

where $E^{i}(P)$ is the true average income in $S_{b_{i}}$, i.e. $E^{i}(P)=E[y \mid y \in$ $\left.S_{b_{i}}\right]{ }^{6}$ Furthermore, the above definition of the belief function implies that misperceptions are constant within groups, i.e. people who are in the same group have the same (mis-)perception about the other groups' average (and thus misperceptions do not depend on one's own income directly, but on group membership). ${ }^{7}$

I also restrict the beliefs of one group about average income in another group to actually lie in that group's income range:

\footnotetext{
${ }^{6}$ For reasons of simplicity, I will restrict attention to partitions $P$ such that all groups $S_{b_{i}}$ have strictly positive measure.

${ }^{7}$ I restrict my attention to misperceptions that are constant within group because I specifically want to focus on differences in perceptions between groups rather than within groups. This restriction helps to simplify the analysis, but the main results of this paper would not change fundamentally if biases were to vary also within groups. The restriction can be deduced "naturally" from the assumption that people interact and communicate freely within their own group and hence will, within their group, reach a common belief about the other groups.
} 
Assumption 1 The belief function $B(P)$ is such that

$$
\inf S_{b_{j}} \leq E_{i}^{j}(P) \leq \sup S_{b_{j}} \forall i \forall j
$$

Given this belief function, I can define the following:

Definition 2 A biased sorting equilibrium is a partition $P=\left[S_{b_{0}}, S_{b_{1}}, \ldots, S_{b_{n}}\right]$ of $Y$ and a menu of sorting fees $\left[b_{0}, b_{1}, . ., b_{n}\right]\left(\right.$ with $b_{i}<b_{i+1} \forall i$ and $b_{0}=0$ ) such that $\forall i \in\{0, . ., n\}$

$$
U\left(y, E^{i}(P)\right)-b_{i} \geq U\left(y, E_{i}^{k}(P)\right)-b_{k} \quad \forall y \in S_{b_{i}}, \forall i, \forall k \neq i .
$$

A biased sorting equilibrium is therefore a partition of $Y$ that is "stable" given people's misperceptions about the other group. When people compare the utility they obtain in their own group and compare it to the utility they think they could obtain in any other group - given their misperceptions about average income in the other groups - they come to the conclusion that they reach the highest possible level of utility in their own group and therefore they do not want to move to another group.

Corollary 4 In any biased sorting equilibrium, groups whose members perceive that the average income in their group is high compared to other groups will have high sorting fees compared to other groups.

Proof. This follows immediately from Definition 2 and the fact that $U$ is strictly increasing.

With biased perceptions, non-monotone sorting equilibria can exist, as the following example demonstrates.

Example 1 Suppose $Y=[0,1]$ and income $y$ is uniformly distributed, and suppose that $U(y, x)=y x$. Suppose the two groups $S_{b_{0}}=\left[0, \frac{1}{4}\right) \cup\left(\frac{1}{2}, \frac{3}{4}\right]$ and $S_{b_{1}}=\left[\frac{1}{4}, \frac{1}{2}\right] \cup\left(\frac{3}{4}, 1\right]$ would constitute an equilibrium partition if people are unbiased. The correct average income of $S_{b_{1}}$ is $\frac{5}{8}$ and the average income of $S_{b_{0}}$ is $\frac{3}{8}$. In an unbiased sorting equilibrium the sorting fee $b_{1}$ must be strictly positive (normalizing $b_{0}=0$ ), because the average in group $S_{b_{1}}$ is higher than in group $S_{b_{0}}$. Now take the person with income $\frac{5}{8}$, who is in 
$S_{b_{0}}$. She derives utility $\frac{5}{8} \times \frac{3}{8}$ from being in $S_{b_{0}}$ and utility $\frac{5}{8} \times \frac{5}{8}-b_{1}$ from being in $S_{b_{1}}$. Because this person is in $S_{b_{0}}$ we must have

$$
\frac{5}{8} \times \frac{5}{8}-b_{1} \leq \frac{5}{8} \times \frac{3}{8}
$$

or equivalently

$$
\frac{5}{8}\left(\frac{5}{8}-\frac{3}{8}\right) \leq b_{1}
$$

At the same time, the person who is at $\frac{3}{8}$ is in $S_{b_{1}}$, hence her utility from being in $S_{b_{1}}$ must be higher than the utility from being in $S_{b_{0}}$. This yields the condition

$$
\frac{3}{8}\left(\frac{5}{8}-\frac{3}{8}\right) \geq b_{1}
$$

It is immediate to see that (4) and (5) contradict each other, so this partition cannot be an unbiased sorting equilibrium. However, depending on the belief function, it is possible that this partition is a biased sorting equilibrium: Suppose that the belief function is such that people in $S_{b_{1}}$ perceive the average in their group and in the other group correctly, but the people in $S_{b_{0}}$ all think that the average in group $S_{b_{1}}$ is equal to the average in their own group. Then they would not be willing to pay any $b_{1}>0$ to join group $S_{b_{1}}$, whereas everybody in group $S_{b_{1}}$ is willing to pay some positive $b_{1}$, e.g. $b_{1}=\frac{1}{16}$ (which makes the poorest person in $S_{b_{1}}$ exactly indifferent between the two groups, while everybody else in $S_{b_{1}}$ strictly prefers being there). In fact any $b_{1} \in\left(0, \frac{1}{16}\right]$ would make the above partition a biased sorting equilibrium.

\subsection{Global and local consistency}

At first, adding misperceptions to the model does not simplify the analysis, but rather adds some additional problems: As the above example shows, with sufficient freedom on how to specify the groups' misperceptions, nonmonotone biased sorting equilibria are possible. I consider this to be an undesirable feature because it complicates the analysis and is at odds with empirical evidence of how groups in society are formed. Additionally, the menu of sorting fees might not be uniquely determined for a given equilibrium partition (see Example 1 above). Moreover, we do not necessarily 
have the notion of consistency (which is inherent in the unbiased model, as explained above) in a biased sorting equilibrium. Go back to Example 1: People's beliefs about the other group are inconsistent with what they see: Everybody in group $S_{b_{0}}$ wonders why anybody would want to pay $b_{1}$ to join $S_{b_{1}}$, while at the same time the people in $S_{b_{1}}$ cannot understand why the people with income between $\frac{1}{2}$ and $\frac{3}{4}$ do not want to join their group.

However, the inconsistency, the non-monotonicity and the non-uniqueness of the menu of sorting fees for a given equilibrium partition vanish if I introduce what I call the consistency requirement. This requirement can hold either locally or (in its stronger version) globally. Let me first introduce the notion of global consistency: This requires that people who are in $S_{b_{i}}$ think that people with different incomes, who are not in $S_{b_{i}}$ but in some other group $S_{b_{j}}$, are correct in doing so. Formally, this requirement translates to

Definition 3 A partition $P=\left[S_{b_{0}}, S_{b_{1}}, . ., S_{b_{n}}\right]$ of $Y$ and a menu of sorting fees $\left[b_{0}, b_{1}, . ., b_{n}\right]$ satisfy global consistency iff $\forall i \in\{0, . ., n\}$

$$
U\left(y, E_{i}^{j}(P)\right)-b_{j} \geq U\left(y, E_{i}^{k}(P)\right)-b_{k} \quad \forall y \in S_{b_{j}}, \forall j, \forall k .
$$

In words, (GC) says that a person in group $S_{b_{i}}$ who looks at a person with income $y$ in any other group $S_{b_{j}}$, thinks that this person cannot achieve higher utility by switching to a different group (and note that the person from group $S_{b_{i}}$ evaluates person $y$ 's utility given her own biased perception of average group incomes $E_{i}$, the one that she has acquired in her group $\left.S_{b_{i}}\right)$.

As I have pointed out, in the "unbiased" sorting equilibrium that I have defined in the previous section, global consistency is implicit. Because everybody has the same (correct) understanding of average incomes in all the groups, people cannot be "puzzled" by other people's choices - everybody evaluates everybody else's utility in the same way. It is only when people have incorrect perceptions of the other groups that consistency becomes a separate issue and is not implicit in the equilibrium definition. People can be happy with their own choices (which means we are in a sorting equilibrium), while at the same time not understanding other people's choices (which means that consistency is violated). Hence, it makes sense - as a refinement to biased sorting equilibria - to define biased sorting 
equilibria which additionally satisfy global consistency:

Definition 4 A biased sorting equilibrium with global consistency is a partition $P=\left[S_{b_{0}}, S_{b_{1}}, . ., S_{b_{n}}\right]$ of $Y$ and a menu of sorting fees $\left[b_{0}, b_{1}, \ldots b_{n}\right]$ $\left(\right.$ with $b_{i}<b_{i+1} \forall i$ and $b_{0}=0$ ) such that $\forall i \in\{0, . ., n\}$

$$
\begin{array}{r}
U\left(y, E^{i}(P)\right)-b_{i} \geq U\left(y, E_{i}^{k}(P)\right)-b_{k} \quad \forall y \in S_{b_{i}}, \forall k \\
U\left(y, E_{i}^{j}(P)\right)-b_{j} \geq U\left(y, E_{i}^{k}(P)\right)-b_{k} \quad \forall y \in S_{b_{j}}, \forall j, \forall k
\end{array}
$$

A less restrictive requirement than global consistency is the notion of local consistency. To explain this concept, I first need to define what neighbouring groups are in the present context:

Definition 5 If $\left[S_{b_{0}}, S_{b_{1}}, \ldots S_{b_{n}}\right]$ is a partition of $Y$ with corresponding sorting fees $\left[b_{0}, b_{1}, \ldots b_{n}\right]\left(\right.$ with $\left.b_{i}<b_{i+1} \forall i\right)$ then for all $i$ the neighbouring groups of $S_{b_{i}}$ are $S_{b_{i-1}}$ and $S_{b_{i+1}}$.

I define neighbouring groups in terms of sorting fees. However, as groups with higher sorting fees correspond to higher average income, this is equivalent to defining them in terms of average income: neighbouring groups are groups which are "next to each other" if ranked according to mean income. Therefore, local consistency can be interpreted in the following way: People understand the decisions of people who have income that is relatively similar to their own income, i.e. people who are in a group that is a bit richer or poorer than their own group. However, they don't think about people who are in much richer or poorer groups (and therefore do not need to think that their decisions are optimal). The reason for why people care only about neighbouring groups could be that these groups are more salient to them, because in effect those are the groups that matter also for their own individual optimal decision-making about which group to join. ${ }^{8}$

\footnotetext{
${ }^{8}$ Another way to think about is that if people only consider members of neighbouring groups, this could be because societal interactions are such that people are - outside of their own group - most likely to interact with members of neighbouring groups, perhaps due to intersecting meeting points of neighbouring groups, such as supermarkets or schools etc.
} 
Local consistency only requires consistency between neighbouring groups and doesn't put any restrictions on what people believe about the optimality of other people's decisions who are not in their neighbouring group. Formally, this is equivalent to

Definition 6 A partition $\left[S_{b_{0}}, S_{b_{1}}, \ldots, S_{b_{n}}\right]$ of $Y$ and a menu of sorting fees $\left[b_{0}, b_{1}, . ., b_{n}\right]$ satisfy local consistency iff $\forall i \in\{0, . ., n\}$

$U\left(y, E_{i}^{j}(P)\right)-b_{j} \geq U\left(y, E_{i}^{k}(P)\right)-b_{k} \quad \forall y \in S_{b_{j}}, \forall k, \forall j \in\{i-1, i+1\} \cap \mathbb{N}$

It is straightforward to see that global consistency is a stricter requirement than local consistency.

Corollary 5 Global consistency implies local consistency.

A biased sorting equilibrium with local consistency is defined as follows:

Definition 7 A biased sorting equilibrium with local consistency is a partition $P=\left[S_{b_{0}}, S_{b_{1}}, \ldots, S_{b_{n}}\right]$ of $Y$ and a menu sorting fees $\left[b_{0}, b_{1}, \ldots b_{n}\right]$ (with $b_{i}<b_{i+1} \forall i$ and $b_{0}=0$ ) such that $\forall i \in\{0, . ., n\}$

$$
U\left(y, E^{i}(P)\right)-b_{i} \geq U\left(y, E_{i}^{k}(P)\right)-b_{k} \geq b_{i}-b_{k} \quad \forall y \in S_{b_{i}}, \forall k
$$

$U\left(y, E_{i}^{j}(P)\right)-b_{j} \geq U\left(y, E_{i}^{k}(P)\right)-b_{k} \quad \forall y \in S_{b_{j}}, \forall k, \forall j \in\{i-1, i+1\} \cap \mathbb{N}$

Requiring (local or global) consistency eliminates non-monotone biased sorting equilibria, as the following example demonstrates:

Example 2 (Example 1 continued) Take again the example from before. I have showed above that the partition $S_{b_{0}}=\left[0, \frac{1}{4}\right) \cup\left(\frac{1}{2}, \frac{3}{4}\right]$ and $S_{b_{1}}=\left[\frac{1}{4}, \frac{1}{2}\right] \cup$ $\left(\frac{3}{4}, 1\right]$ together with $b_{1}=\frac{1}{16}, b_{0}=0, E_{1}^{1}(P)=E^{1}(P), E_{1}^{0}(P)=E^{0}(P)$, $E_{0}^{1}(P)=E_{0}^{0}(P)=E^{0}(P)$ is a biased sorting equilibrium. However, local consistency ${ }^{9}$ does not hold here: All people in $S_{b_{0}}$ think that the people in

\footnotetext{
${ }^{9}$ Both global and local consistency do not hold in this case, because in the case of two groups these two notions are identical.
} 
$S_{b_{1}}$ are wrong to pay $b_{1}$ and join $S_{b_{1}}$, and equally all people in $S_{b_{1}}$ do not understand why the people with $y \in\left(\frac{1}{2}, \frac{3}{4}\right)$ do not want to join $S_{b_{1}}$.

The finding that non-monotone biased sorting equilibria do not satisfy local consistency can be generalized.

Proposition 2 All biased sorting equilibria with local consistency satisfy monotonicity.

Proof. Suppose a non-monotone equilibrium exists. Then there must be two neighbouring groups $S_{b_{i}}$ and $S_{b_{i-1}}$ with $y_{2} \in S_{b_{i-1}}$ and $y_{1} \in S_{b_{i}}$ but $y_{2}>y_{1}$. Then $(\mathrm{IC})$ requires

$$
b_{i}-b_{i-1} \geq U\left(y_{2}, E_{i-1}^{i}(P)\right)-U\left(y_{2}, E_{i-1}^{i-1}(P)\right)
$$

and

$$
U\left(y_{1}, E_{i}^{i}(P)\right)-U\left(y_{1}, E_{i}^{i-1}(P)\right) \geq b_{i}-b_{i-1} .
$$

These two conditions combined give

$$
U\left(y_{1}, E_{i}^{i}(P)\right)-U\left(y_{1}, E_{i}^{i-1}(P)\right) \geq U\left(y_{2}, E_{i-1}^{i}(P)\right)-U\left(y_{2}, E_{i-1}^{i-1}(P)\right)
$$

and as $E_{i-1}$ and $E_{i}$ can be chosen freely, this inequality can hold for some $E_{i}$ and $E_{i-1}$, even though $y_{1}<y_{2}$. However, the consistency requirement (LC) yields the additional conditions

$$
U\left(y_{1}, E_{i-1}^{i}(P)\right)-U\left(y_{1}, E_{i-1}^{i-1}(P)\right) \geq b_{i}-b_{i-1}
$$

and

$$
b_{i}-b_{i-1} \geq U\left(y_{2}, E_{i}^{i}(P)\right)-U\left(y_{2}, E_{i}^{i-1}(P)\right) .
$$

But (7) and (9) together imply

$$
U\left(y_{1}, E_{i}^{i}(P)\right)-U\left(y_{1}, E_{i}^{i-1}(P)\right) \geq U\left(y_{2}, E_{i}^{i}(P)\right)-U\left(y_{2}, E_{i}^{i-1}(P)\right)
$$

and (6) and (8) together imply

$$
U\left(y_{1}, E_{i-1}^{i}(P)\right)-U\left(y_{1}, E_{i-1}^{i-1}(P)\right) \geq U\left(y_{2}, E_{i-1}^{i}(P)\right)-U\left(y_{2}, E_{i-1}^{i-1}(P)\right) .
$$


Neither of these inequalities can hold if $y_{2}>y_{1}$ and $U$ is strictly supermodular.

Proposition 2 shows that we do not need to assume global consistency to get monotonicity, local consistency is enough to ensure that even if people are biased there cannot be any other equilibria apart from monotone ones. Hence, global and local consistency are equivalent In terms of guaranteeing monotone equilibria. However, the difference between local and global consistency will play an important role in Section 6, when I analyze existence of biased sorting equilibria with more than two groups.

Proposition 2 implies that all biased sorting equilibria with consistency (both local and/or global) can be characterized in terms of cutoffs $\hat{y}_{i}$ and biased perceptions $E_{i}$.

Corollary 6 A biased sorting equilibrium with global consistency is characterized by a partition $\left[0, \hat{y}_{1}, \ldots, \hat{y}_{n}, y_{\max }\right]$ of $Y$ and a menu of sorting fees $\left[b_{0}, b_{1}, . ., b_{n}\right]$ (with $b_{i}<b_{i+1} \forall i$ and $\left.b_{0}=0\right)$ such that $\forall i \in\{1, . ., n\}$

$$
\begin{gathered}
U\left(\hat{y}_{i}, E_{0}^{i}(P)\right)-U\left(\hat{y}_{i}, E_{0}^{i-1}(P)\right) \\
=U\left(\hat{y}_{i}, E_{1}^{i}(P)\right)-U\left(\hat{y}_{i}, E_{1}^{i-1}(P)\right) \\
=\ldots=U\left(\hat{y}_{i}, E_{n}^{i}(P)\right)-U\left(\hat{y}_{i}, E_{n}^{i-1}(P)\right) \\
=b_{i}-b_{i-1} \quad \forall i \in\{1, . ., n\}
\end{gathered}
$$

Corollary 7 A biased sorting equilibrium with local consistency is characterized by a partition $\left[0, \hat{y}_{1}, \ldots, \hat{y}_{n}, y_{\max }\right]$ of $Y$ and a menu of sorting fees $\left[b_{0}, b_{1}, . ., b_{n}\right]\left(\right.$ with $b_{i}<b_{i+1} \forall i$ and $\left.b_{0}=0\right)$ such that $\forall i \in\{1, . ., n\}$

$$
\begin{gathered}
U\left(\hat{y}_{i}, E^{i}(P)\right)-U\left(\hat{y}_{i}, E_{i}^{i-1}(P)\right) \\
=U\left(\hat{y}_{i}, E_{i-1}^{i}(P)\right)-U\left(\hat{y}_{i}, E^{i-1}(P)\right) \\
=b_{i}-b_{i-1}
\end{gathered}
$$


and $d^{10}$

$$
U\left(y, E^{i}(P)\right)-b_{i} \geq U\left(y, E_{i}^{k}(P)\right)-b_{k} \quad \forall y \in S_{b_{i}}, \forall k \neq i
$$

Proof. (IC) and (LC) imply that

$$
\begin{array}{r}
U\left(y, E^{i}(P)\right)-b_{i} \geq U\left(y, E_{i}^{i-1}(P)\right)-b_{i-1} \quad \forall y \in S_{b_{i}}, \forall i \\
\left.\Longleftrightarrow U\left(y, E^{i}(P)\right]\right)-U\left(y, E_{i}^{i-1}(P)\right) \geq b_{i}-b_{i-1} \quad \forall y \in S_{b_{i}}, \forall i
\end{array}
$$

and

$$
\begin{array}{r}
U\left(y, E^{i}(P)\right)-b_{i} \leq U\left(y, E_{i}^{i-1}(P)\right)-b_{i-1} \quad \forall y \in S_{b_{i-1}}, \forall i \\
\Longleftrightarrow U\left(y, E^{i}(P)\right)-U\left(y, E_{i}^{i-1}(P)\right) \leq b_{i}-b_{i-1} \quad \forall y \in S_{b_{i-1}}, \forall i
\end{array}
$$

(13) and (14) together with the fact that $S_{b_{i}}=\left[\hat{y}_{i}, \hat{y}_{i+1}\right]$ and $S_{b_{i-1}}=\left[\hat{y}_{i-1}, \hat{y}_{i}\right]$ imply that

$$
U\left(\hat{y}_{i}, E^{i}(P)\right)-U\left(\hat{y}_{i}, E_{i}^{i-1}(P)\right)=b_{i}-b_{i-1} \quad \forall i
$$

Furthermore, (IC) and (LC) also imply that

$$
\begin{aligned}
U\left(y, E_{i-1}^{i}(P)\right)-b_{i} \geq U\left(y, E^{i-1}(P)\right)-b_{i-1} \quad \forall y \in S_{b_{i}}, \forall i \\
\Longleftrightarrow U\left(y, E_{i-1}^{i}(P)\right)-U\left(y, E^{i-1}(P)\right) \geq b_{i}-b_{i-1} \quad \forall y \in S_{b_{i}}, \forall i
\end{aligned}
$$

and

$$
\begin{array}{r}
U\left(y, E_{i-1}^{i}(P)\right)-b_{i} \leq U\left(y, E^{i-1}(P)\right)-b_{i-1} \quad \forall y \in S_{b_{i-1}}, \forall i \\
\Longleftrightarrow U\left(y, E_{i-1}^{i}(P)\right)-U\left(y, E^{i-1}(P)\right) \leq b_{i}-b_{i-1} \quad \forall y \in S_{b_{i-1}}, \forall i
\end{array}
$$

(16) and (17) together with $S_{b_{i}}=\left[\hat{y}_{i}, \hat{y}_{i+1}\right]$ and $S_{b_{i-1}}=\left[\hat{y}_{i-1}, \hat{y}_{i}\right]$ imply that

$$
U\left(\hat{y}_{i}, E_{i-1}^{i}(P)\right)-U\left(\hat{y}_{i}, E^{i-1}(P)\right)=b_{i}-b_{i-1} \quad \forall i
$$

Combined, (15) and (18) give the equilibrium condition (11).

Hence, a biased sorting equilibrium with local consistency is a partition where the perceived benefit of being in group $S_{b_{i}}$ rather than $S_{b_{i-1}}$

\footnotetext{
${ }^{10}$ Condition (11) ensures that condition (12) holds for $k \in\{i-1, i+1\}$ but we need to ensure that people prefer their group $S_{b_{i}}$ to any other group $S_{b_{k}}$. This condition is not needed for the definition of biased sorting equilibrium with global consistency, because it is guaranteed by condition (10).
} 
of the person just to the right of every cutoff $\hat{y}_{i}$ (which is $U\left(\hat{y}_{i}, E^{i}(P)\right)-$ $\left.U\left(\hat{y}_{i}, E_{i}^{i-1}(P)\right)\right)$ is the same as the perceived benefit of being in group $S_{b_{i}}$ rather than $S_{b_{i-1}}$ of the person just to the left of every cutoff $\hat{y}_{i}\left(U\left(\hat{y}_{i}, E_{i-1}^{i}(P)\right)-\right.$ $\left.U\left(\hat{y}_{i}, E^{i-1}(P)\right)\right)$. The equilibrium conditions (11) and (12) restrict the sets of belief functions which imply equilibrium existence. In the next sections, I put more structure on the functional form of the belief functions and find conditions on these functions such that equilibrium exists and is unique. If the misperceptions are such that the biased sorting equilibrium partition with consistency is unique, it follows from (11) that the corresponding schedule of sorting fees will also be unique (as long as $b_{0}$ is normalized to $0)$.

\section{Existence and uniqueness of binary biased sorting equilibria with consistency}

What kind of biased sorting equilibria can exist for different types of misperceptions? In the following section, I will focus on (monotone) partitions with two groups $P=\left[S_{0}, S_{b}\right]$ of $Y$ that can be uniquely characterized by a cutoff $\hat{y} \in Y$ (with the convention that $S_{0}=[0, \hat{y})$ and $S_{b}=\left[\hat{y}, y_{\max }\right]$ ). I will henceforth call the people in $S_{0}$ "the poor" and the people in $S_{b}$ "the rich".

The belief function $B$ in the two-group case is a continuous function that maps all monotone partitions of $Y$ (and note that any monotone partition can be uniquely characterized by the cutoff $\hat{y}$ ) into a four-dimensional vector of beliefs

$$
B(\hat{y})=\left(\underline{E}(\hat{y}), \bar{E}_{p}(\hat{y}), \underline{E}_{r}(\hat{y}), \bar{E}(\hat{y})\right)
$$

where the first two entries denote the poor group's belief about average income in the poor and the rich group respectively and the last two entries denote the rich group's belief about average income in the poor and the rich group. $\underline{E}(\hat{y})$ is the true average income in the poor group, i.e. $\underline{E}(\hat{y})=$ $E[y \mid y<\hat{y}]$ and $\bar{E}(\hat{y})$ is the correct average income in the rich group, $\bar{E}(\hat{y})=$ $E[y \mid y \geq \hat{y}]$. The poor's belief about average income in the rich group is $\bar{E}_{p}(\hat{y})$ and the rich's belief about average income in the poor group is $\underline{E}_{r}(\hat{y})$.

In the following analysis I will restrict my attention to misperceptions 
where the direction of the bias does not vary with the cutoff, i.e. for any configuration of groups one group either always overestimates or underestimates average income in the other group and groups do not switch between over- and underestimating each other depending on group size or shape. Formally, this means I look at belief functions $B$ that satisfy

$$
\bar{E}_{p}(\hat{y})<(>) \bar{E}(\hat{y}) \quad \forall \hat{y} \in\left[0, y_{\max }\right)
$$

and

$$
\underline{E}_{r}(\hat{y})>(<) \underline{E}(\hat{y}) \quad \forall \hat{y} \in\left(0, y_{\max }\right] .
$$

Note, though, that by Assumption 1 a group is correct about average income in the other group if they see the whole income distribution, i.e. the poor are correct at $y_{\max }$,

$$
\bar{E}_{p}(\hat{y})=\bar{E}(\hat{y}) \text { if } \hat{y}=y_{\max }
$$

and the rich are correct at 0 ,

$$
\underline{E}_{r}(\hat{y})=\underline{E}(\hat{y}) \quad \text { if } \hat{y}=0 \text {. }
$$

Then there are four possible combinations of biases:

Case 1: $\underline{E}_{r}(\hat{y})>\underline{E}(\hat{y})$ and $\bar{E}_{p}(\hat{y})<\bar{E}(\hat{y}) \quad \forall \hat{y} \in\left(0, y_{\max }\right)$ : The rich overestimate average income in the poor group and the poor underestimate average income in the rich group.

Case 2: $\underline{E}_{r}(\hat{y})<\underline{E}(\hat{y})$ and $\bar{E}_{p}(\hat{y})>\bar{E}(\hat{y}) \quad \forall \hat{y} \in\left(0, y_{\max }\right)$ : The rich underestimate average income in the poor group and the poor overestimate average income in the rich group.

Case 3: $\underline{E}_{r}(\hat{y})<\underline{E}(\hat{y})$ and $\bar{E}_{p}(\hat{y})<\bar{E}(\hat{y}) \forall \hat{y} \in\left(0, y_{\max }\right)$ : Both groups underestimate each other's average income

Case 4: $\underline{E}_{r}(\hat{y})>\underline{E}(\hat{y})$ and $\bar{E}_{p}(\hat{y})>\bar{E}(\hat{y}) \forall \hat{y} \in\left(0, y_{\max }\right)$ : Both groups overestimate each other's income.

In the first case, both groups underestimate the difference between groups, while in the second case they both overestimate it. In the latter 
two cases the misperceptions work in opposite directions for the two groups: one group overestimates the difference, the other group underestimates it. In Appendix 8.1 I analyze these four combinations to see whether biased sorting equilibria (with and without consistency) can exist and I provide sufficient conditions for existence and uniqueness. (Note that global and local consistency are the same concept in the case of two groups, which is why I talk only about "consistency" in this section.) It turns out that interior biased sorting equilibria with consistency can only exist in two of the four possible combinations: either both groups think the other group is more similar to themselves or both groups think the other group is more different to themselves. The reason for this is that the equilibrium condition (11) requires both groups to have the same perception of the benefits of sorting at the equilibrium cutoff, and hence it cannot be the case that one group underestimates the difference between the groups for any cutoff, while the other group overestimates it.

Proposition 3 No cutoff $\hat{y} \in Y$ constitutes a biased sorting equilibrium in Case 4.

Proposition 4 Any cutoff $\hat{y} \in Y$ constitutes a biased sorting equilibrium in Case 3, but no cutoff $\hat{y} \in Y$ constitutes a biased sorting equilibrium with consistency.

Proof. See Appendix 8.1.

While biased sorting equilibria with consistency cannot exist in Case 3 and 4, it turns out that they will always exist in Case 1 and 2.

Proposition 5 There always exists a biased sorting equilibrium with consistency in Case 1 and 2.

Proof. See Appendix 8.1.

The intuition for why Case 1- and Case 2-type misperceptions guarantee the existence of equilibria is that the form of the misperceptions ensures that the groups' perceived benefits of sorting cross at least once: At $\hat{y}=0$ the poor's perceived benefits of sorting are lower (higher) than the rich's, and the reverse is true at $\hat{y}=y_{\max }$. As all functions are continuous, there must be an interior cutoff $\hat{y} \in\left(0, y_{\max }\right)$ such that they are equal. 
If $B$ and $U(.,$.$) are such that the perceived benefits of sorting of the$ two groups intersect only once, the interior equilibrium cutoff is unique. Sufficient conditions for this are stated in Appendix 8.1.3. If $U$ is linear in both arguments ${ }^{11}$, the sufficient conditions for uniqueness simplify to the following:

Proposition 6 (Case 1 Uniqueness) If $U(.,$.$) is linear and people are bi-$ ased according to Case 1, the biased sorting equilibrium with consistency is unique if the misperceptions monotonically converge to the truth, i.e.

$$
\frac{d\left(\bar{E}(\hat{y})-\bar{E}_{p}(\hat{y})\right)}{d \hat{y}}<0 \quad \text { and } \quad \frac{d\left(\underline{E}_{r}(\hat{y})-\underline{E}(\hat{y})\right)}{d \hat{y}}>0 \quad \forall \hat{y} \in\left(0, y_{\max }\right)
$$

Proof. See Appendix 8.1.1.

Proposition 7 (Case 2 Uniqueness) If $U(.,$.$) is linear and people are bi-$ ased according to Case 2, the interior equilibrium is unique if the misperceptions monotonically converge to the truth, i.e.

$$
\frac{d\left(\bar{E}_{p}(\hat{y})-\bar{E}(\hat{y})\right)}{d \hat{y}}<0 \quad \text { and } \quad \frac{d\left(\underline{E}(\hat{y})-\underline{E}_{r}(\hat{y})\right)}{d \hat{y}}>0 \quad \forall \hat{y} \in\left(0, y_{\max }\right)
$$

Proof. See Appendix 8.1.1.

The condition that the misperceptions converge to the truth monotonically as the cutoff goes to 0 resp. $\hat{y}$, i.e.

$$
\frac{d\left|\bar{E}(\hat{y})-\bar{E}_{p}(\hat{y})\right|}{d \hat{y}}<0 \text { and } \frac{d\left|\underline{E}_{r}(\hat{y})-\underline{E}(\hat{y})\right|}{d \hat{y}}>0
$$

can be interpreted as people being less biased, the more they see of the income distribution: as $\hat{y}$ increases, the poor see a bigger part of the income distribution and their belief about average income in the other group becomes more accurate. The opposite happens for the rich: as the cutoff increases, they see a smaller part of society and therefore become more biased. In the limit this yields what I have already assumed in Assumption 1: the poor are correct at $y_{\max }$ and the rich at 0 .

\footnotetext{
${ }^{11}$ If $U$ is linear then it is not strictly increasing whenever one of the arguments is 0 . See Appendix 8.1.1 for a specific analysis of linear utility functions at 0 .
} 


\section{Existence of biased sorting equilibria with consistency and more than two groups}

In the following section, I will assume that the utility function $U(.,$.$) is lin-$ ear in both arguments, because it greatly simplifies my analysis. However, the results would not change qualitatively for a general strictly increasing and strictly supermodular utility function.

Whether biased sorting equilibria with consistency can exist and will be unique in the case of more than two groups depends, as in the two-group case, on the belief function. In addition, existence and uniqueness depend in general on the underlying income distribution. Finally, for more than two groups it also matters whether we want to require local or global consistency (while in the two-group case those two concepts are the same). For example, if the belief function is such that the perceived difference between group average incomes decreases in the distance (in terms of average income) of one's own group to the observed groups (perhaps because all groups that are far away from one's own group appear roughly similar), no equilibrium with more than two groups can exist if global consistency is required. The reason is that under global consistency, perceived differences between groups have to be the same across groups. For example, in the case of three groups the equilibrium conditions for a sorting equilibrium with global consistency would be ${ }^{12}$

$$
E_{0}^{1}-E_{0}^{0}=E_{1}^{1}-E_{1}^{0}=E_{2}^{1}-E_{2}^{0}
$$

and

$$
E_{0}^{2}-E_{0}^{1}=E_{1}^{2}-E_{1}^{1}=E_{2}^{2}-E_{2}^{1} .
$$

If the perceived difference in group averages is decreasing with distance, these equalities obviously cannot hold (for example, we would have $E_{0}^{2}-$ $E_{0}^{1}<E_{1}^{2}-E_{1}^{1}$ etc.). The same problem would occur for four or more groups. Hence, biased sorting equilibria with global consistency and more than two groups are not possible if the belief function specifies decreasing perceived group average differences. Of course, the same result holds for

\footnotetext{
${ }^{12}$ To improve readability I will from now on write $E_{i}^{j}$ instead of $E_{i}^{j}(P)$, but the group beliefs continue to be functions of the partition.
} 
belief functions with increasing perceived group average differences.

On the other hand, biased sorting equilibria with local consistency are still possible with the above beliefs, because non-neighbouring groups don't matter in this case. However, it is important that neighbouring groups assess the differences between each other in the same way, so beliefs cannot be such that one group overestimates the difference to a neighbouring group, while this neighbouring group underestimates it.

In the following analysis I want to focus on a particular form of beliefs: belief functions which are such that misperceptions about a group's average income do not depend on whether this group is a neighbouring group or not, and thus the severity of the bias depends only on group size, irrespective of which group people look at (and so e.g. people in the poorest group are "equally" biased about the average income in all other groups, no matter whether it is their neighbouring group or a very rich group). Specifically, I will for the rest of this section focus on beliefs that are of a dertain functional form, which I will call Proportional Biased Beliefs. Proportional Biased Beliefs of Type 1 are such that all groups underestimate the differences between their own group and other groups, whereas Proportional Biased Beliefs of Type 2 are such that all groups overestimate the differences between their own group and other groups.

Definition 8 A belief function $B(P)$ generates Proportional Biased Beliefs of Type 1 if group $i$ 's belief about average income in group $j<i$ are ${ }^{13}$

$$
E_{i}^{j}=\beta\left(1-F_{i+1}+F_{i}\right) \hat{y}_{j+1}+\left(1-\beta\left(1-F_{i+1}+F_{i}\right)\right) E^{j} \forall i \forall j
$$

and group i's beliefs about average income in group $k>i$ are

$$
E_{i}^{k}=\beta\left(1-F_{i+1}+F_{i}\right) \hat{y}_{k}+\left(1-\beta\left(1-F_{i+1}+F_{i}\right)\right) E^{k} \forall i \forall k .
$$

Definition 9 A belief function $B(P)$ generates Proportional Biased Beliefs of Type 2 if group $i$ 's belief about average income in group $j<i$ are

$$
E_{i}^{j}=\beta\left(1-F_{i+1}+F_{i}\right) \hat{y}_{j}+\left(1-\beta\left(1-F_{i+1}+F_{i}\right)\right) E^{j} \forall i \forall j
$$

\footnotetext{
${ }^{13}$ To improve readability I will from now on write $F_{i}$ instead of $F\left(\hat{y}_{i}\right)$.
} 
and group i's beliefs about average income in group $k>i$ are

$$
E_{i}^{k}=\beta\left(1-F_{i+1}+F_{i}\right) \hat{y}_{k+1}+\left(1-\beta\left(1-F_{i+1}+F_{i}\right)\right) E^{k} \forall i \forall k .
$$

Remark 1 Note that by "group $i$ "I mean people in group $S_{b_{i}}$, i.e. people with income between cutoffs $\hat{y}_{i}$ and $\hat{y}_{i+1}$. Average income between $y_{i}$ and $y_{i+1}$ is denoted as $E^{i}$.

With Proportional Biased Beliefs of Type 1 (Type 2), people tend to underestimate (overestimate) differences between their own group and other groups (in terms of average group income), and their misperceptions are more severe, the smaller their group (i.e. the less they see of the whole income distribution).

These beliefs satisfy the necessary and sufficient conditions for existence and uniqueness of binary biased sorting equilibria as stated in Section 5: They are either Case 1- or Case 2- type misperceptions and satisfy Assumption 1. In Appendix 8.2.3 I show that with Proportional Biased Beliefs, biased sorting equilibria with global consistency do not exist for more than three groups. The reason for this is of a technical nature: With global consistency and this particular specification of the bias, everybody must have the same perceived benefits of being in one group versus being in another group. However, as everybody knows the average income in their own group, but misperceives average incomes in the other groups, this creates a difference between perceived benefits of being in one's own group versus being in any other group, and perceived benefits of being in one group versus another group while not being a member of either group. As these different perceived benefits have to be the same across groups, this yields a contradiction. For three groups, existence with global consistency and the above defined bias depends on the underlying distribution. For instance, if the income distribution is uniform, an equilibrium partition with three groups cannot exist, as shown below.

In contrast, it turns out that with the same belief function, there can be more than three groups if only local consistency is required. For example, if the income distribution is uniform, any partition with equidistant (finitely many) cutoffs is a biased sorting equilibrium with local consistency. I prove this in Appendix 8.2.1. 
Let me illustrate these findings with a simple example: Suppose $y$ is uniformly distributed on $[0, a]$. A biased sorting equilibrium with local consistency and three groups needs to satisfy the following equilibrium conditions

$$
\begin{aligned}
& E_{0}^{1}-E_{0}^{0}=E_{1}^{1}-E_{1}^{0} \\
& E_{1}^{2}-E_{1}^{1}=E_{2}^{2}-E_{2}^{1}
\end{aligned}
$$

and

$$
\begin{aligned}
& \hat{y}_{1}\left(E_{0}^{2}-E_{0}^{0}\right) \leq b_{2} \\
& \hat{y}_{2}\left(E^{2}-E_{2}^{0}\right) \geq b_{2}
\end{aligned}
$$

with

$$
b_{2}=\hat{y}_{2}\left(E^{2}-E_{2}^{1}\right)+b_{1}=\hat{y}_{2}\left(E_{1}^{2}-E^{1}\right)+b_{1}
$$

and

$$
b_{1}=\hat{y}_{1}\left(E^{1}-E_{1}^{0}\right)=\hat{y}_{1}\left(E_{0}^{1}-E^{0}\right) .
$$

Suppose that people have proportional biased beliefs of type 1, i.e.

$$
\begin{aligned}
& E_{0}^{0}=E^{0} \\
& E_{0}^{1}=\beta\left(1-F\left(\hat{y}_{1}\right)\right) \hat{y}_{1}+\left(1-\beta\left(1-F\left(\hat{y}_{1}\right)\right)\right) E^{1} \\
& E_{0}^{2}=\beta\left(1-F\left(\hat{y}_{1}\right)\right) \hat{y}_{2}+\left(1-\beta\left(1-F\left(\hat{y}_{1}\right)\right)\right) E^{2} \\
& E_{1}^{0}=\beta\left(1-F\left(\hat{y}_{2}\right)+F\left(\hat{y}_{1}\right)\right) \hat{y}_{1}+\left(1-\beta\left(1-F\left(\hat{y}_{2}\right)+F\left(\hat{y}_{1}\right)\right)\right) E^{0} \\
& E_{1}^{1}=E^{1} \\
& E_{1}^{2}=\beta\left(1-F\left(\hat{y}_{2}\right)+F\left(\hat{y}_{1}\right)\right) \hat{y}_{2}+\left(1-\beta\left(1-F\left(\hat{y}_{2}\right)+F\left(\hat{y}_{1}\right)\right)\right) E^{2} \\
& E_{2}^{0}=\beta F\left(\hat{y}_{2}\right) \hat{y}_{1}+\left(1-\beta F\left(\hat{y}_{2}\right)\right) E^{0} \\
& E_{2}^{1}=\beta F\left(\hat{y}_{2}\right) \hat{y}_{2}+\left(1-\beta F\left(\hat{y}_{2}\right)\right) E^{1} \\
& E_{2}^{2}=E^{2}
\end{aligned}
$$

Given the functional form of the misperceptions, the equilibrium conditions (19) and (20) yield

$$
\hat{y}_{1}=\frac{\left(1-F_{2}+F_{1}\right) E^{0}+\left(1-F_{1}\right) E^{1}}{2-F_{2}}
$$


and

$$
\hat{y}_{2}=\frac{F_{2} E^{1}+\left(1-F_{2}+F_{1}\right) E^{2}}{1+F_{1}}
$$

Using the fact that $F$ is uniformly distributed on $[0, a]$ yields the unique solution

$$
\begin{aligned}
& \hat{y}_{1}=\frac{a}{3} \\
& \hat{y}_{2}=\frac{2 a}{3} .
\end{aligned}
$$

Note that this implies that in equilibrium the three groups will all be of equal size, i.e.

$$
F_{1}=F_{2}-F_{1}=1-F_{2}=\frac{1}{3} .
$$

Also conditions (21) and (22) are satisfied in this case: with equidistant cutoffs and a uniform distribution we get

$$
b_{1}=\left(\frac{a}{3}\right)^{2}\left[1-\beta \frac{1}{3}\right]
$$

and

$$
b_{2}=3\left(\frac{a}{3}\right)^{2}\left[1-\beta \frac{1}{3}\right]=3 b_{1}
$$

and hence condition (21) becomes

$$
\frac{a}{3}\left[\frac{5 a}{6}-\beta \frac{2}{3} \frac{a}{6}-\frac{a}{6}\right] \leq 3\left(1-\beta \frac{1}{3}\right)\left(\frac{a}{3}\right)^{2}
$$

which reduces to

$$
\beta \frac{2}{3} \leq 1
$$

and is therefore always satisfied. Condition (22) becomes

$$
\frac{2 a}{3}\left[\frac{5 a}{6}-\frac{a}{6}-\beta \frac{2}{3} \frac{a}{6}\right] \geq 3\left(1-\beta \frac{1}{3}\right)\left(\frac{a}{3}\right)^{2}
$$

which reduces to

$$
\beta \frac{1}{3} \geq-1
$$

which always holds. Hence, for the uniform distribution there exists a unique biased sorting equilibrium with local consistency and three groups 
if the misperceptions are as defined above.

However, with those same misperceptions, there doesn't exist a sorting equilibrium with three groups if global consistency is required. Global consistency yields the equilibrium conditions

$$
E_{0}^{1}-E_{0}^{0}=E_{1}^{1}-E_{1}^{0}=E_{2}^{1}-E_{2}^{0}
$$

and

$$
E_{0}^{2}-E_{0}^{1}=E_{1}^{2}-E_{1}^{1}=E_{2}^{2}-E_{2}^{1} .
$$

If $F$ is uniform, this translates to the following four conditions:

$$
\begin{gathered}
E^{1}+\frac{\left(2 F_{2}-F_{1}-1\right)}{F_{2}}\left(\hat{y}_{1}-E^{0}\right)=\hat{y}_{2} \\
\frac{2 F_{1}-F_{2}}{1-F_{1}}\left(E^{2}-\hat{y}_{2}\right)+E^{1}=\hat{y}_{1} \\
\hat{y}_{1}=\frac{\left(1-F_{2}+F_{1}\right) E^{0}+\left(1-F_{1}\right) E^{1}}{2-F_{2}} \\
\hat{y}_{2}=\frac{F_{2} E^{1}+\left(1-F_{2}+F_{1}\right) E^{2}}{1+F_{1}}
\end{gathered}
$$

Conditions (27) and (28) are the same as for the equilibrium with local consistency, and we know they yield the unique solution

$$
\begin{aligned}
& \hat{y}_{1}=\frac{a}{3} \\
& \hat{y}_{2}=\frac{2 a}{3}
\end{aligned}
$$

with groups of equal size. However, from (25) it follows that

$$
2 F_{2}-F_{1}-1>0
$$

in equilibrium, because $y_{2}$ must be greater than $E^{1}$. This can be rewritten as

$$
F_{2}-F_{1}>1-F_{2},
$$

which is a contradiction to groups being of equal size. Therefore, no biased 
sorting equilibrium with global consistency and three groups can exist for the uniform distribution if misperceptions are defined as above.

\section{Conclusion}

In this paper I have introduced a new framework for modelling situations in which beliefs and group choice interact endogenously: a model of sorting in the presence of misperceptions that takes into account the two-way interaction between beliefs about society and social segregation. Furthermore, I have defined a new equilibrium concept, the biased sorting equilibrium, which can be interpreted as characterizing partitions which are stable given the misperceptions that people acquire once they are segregated and interact only with members of their own social group. I have also introduced a refinement concept, the consistency requirement, which can be stated in a local and a global version, and I have showed that it guarantees that the biased sorting equilibrium partitions will be monotone. It also adds structure to the model: not every monotone partition of the income space can constitute a biased sorting equilibrium with consistency - existence and uniqueness of equilibrium will depend on the functional form that is used to model people's beliefs about the other groups. For the two-group case I have proved that the consistency requirement guarantees uniqueness of the equilibrium partition if people's misperceptions are such that they converge to the truth monotonically as the size of their group increases. In the case of multiple groups, the main contribution of this paper is to demonstrate that existence of equilibria depends both on the functional form of the belief function and on what type of consistency (local or global) is required. Focusing on a specific functional form of the misperceptions, which I call Proportional Biased Beliefs, I have demonstrated that global consistency precludes existence of equilibria with more than two groups and I have examined conditions for equilibria with local consistency. My findings in this section show that the types of stable partitions that are possible if we allow for more than two groups in equilibrium depend on the situation we want to model, and how we assume the sorting process to look like.

To conclude, let me note that the framework that I have developed in this paper is very general and lends itself to many further applications. In 
the present paper, the variable according to which people sort is income, but any other variable where everybody in society agrees that "more is better" would work as well. For instance, if we want to examine school choice, the sorting variable could be "ability" or "intelligence". With a different utility function (e.g. one that accounts for homophily), the same framework could be applied to model ethnic, religious or cultural segregation.

\section{References}

[1] Abdulkadiroğlu, A., Angrist, J., \& Pathak, P. (2014). The elite illusion: Achievement effects at Boston and New York exam schools. Econometrica, 82(1), 137-196.

[2] Alesina, A., \& Angeletos, G. M. (2005). Fairness and Redistribution. American Economic Review, 960-980.

[3] Algan, Y., Do, Q. A., Dalvit, N., Le Chapelain, A., \& Zenou, Y. (2015). How Social Networks Shape Our Beliefs: A Natural Experiment among Future French Politicians. (mimeo)

[4] Andreasch, M., Fessler, P., Mooslechner, P., Schürz, M. (2013) Fakten zur Vermögensverteilung in Österreich, BMASK (Ed.), Sozialbericht 2011-2012 (Vienna 2013) 247-265.

[5] Ashok, V., Kuziemko, I., \& Washington, E. (2015). Support for Redistribution in an Age of Rising Inequality:: New stylized facts and some tentative explanations. Brookings papers on economic activity, (1), 2.

[6] Bagwell, L. S., \& Bernheim, B. D. (1996). Veblen effects in a theory of conspicuous consumption. The American Economic Review, 349-373.

[7] Bartels, L. M. (2009). Economic inequality and political representation. The unsustainable American state, 167-96.

[8] Becker, G. S. (1974). A theory of marriage. In Economics of the family: Marriage, children, and human capital (pp. 299-351). University of Chicago Press. 
[9] Benabou, R., \& Ok, E. A. (2001). Social Mobility and the Demand for Redistribution: The Poum Hypothesis. The Quarterly Journal of Economics, 116(2), 447-487.

[10] Bishop, B. (2008). The big sort. Why the Clustering of America is Tearing Us Apart. NY: Houghton Mifflin.

[11] Boisjoly, J., Duncan, G. J., Kremer, M., Levy, D. M., \& Eccles, J. (2006). Empathy or antipathy? The impact of diversity. The American economic review, 96(5), 1890-1905.

[12] Bonica, A., McCarty, N., Poole, K. T., \& Rosenthal, H. (2013). Why hasn't democracy slowed rising inequality?. The Journal of Economic Perspectives, 27(3), 103-123.

[13] Borge, L., \& Rattsoe, E. J. (2004). Income Distribution and Tax Structure: Empirical Test of the Meltzer Richard Hypothesis. European Economic Review, 48, 805-826.

[14] Burns, J., Corno, L., \& La Ferrara, E. (2013). Does interaction affect racial prejudice and cooperation? Evidence from randomly assigned peers in South Africa. (mimeo)

[15] Chetty, R., Hendren, N., Kline, P., \& Saez, E. (2014). Where is the land of opportunity? The geography of intergenerational mobility in the United States (No. w19843).

[16] Corneo, G., \& Grüner, H. P. (2000). Social limits to redistribution. The American Economic Review, 90(5), 1491-1507.

[17] Cowell, F. A. (2000). Measurement of inequality. Handbook of income distribution, 1, 87-166.

[18] Cowell, F. (2011). Measuring inequality. OUP Oxford.

[19] Cruces, G., Perez-Truglia, R., \& Tetaz, M. (2013). Biased perceptions of income distribution and preferences for redistribution: Evidence from a survey experiment. Journal of Public Economics, 98, 100-112. 
[20] Dalton, H. (1920). The measurement of the inequality of incomes. The Economic Journal, 30(119), 348-361.

[21] Damiano, E., \& Li, H. (2007). Price discrimination and efficient matching. Economic Theory, 30(2), 243-263.

[22] Dustmann, C., \& Preston, I. (2001). Attitudes to ethnic minorities, ethnic context and location decisions. The Economic Journal, 111(470), 353-373.

[23] Epple, D., \& Romano, R. E. (1998). Competition between private and public schools, vouchers, and peer-group effects. American Economic Review, 33-62.

[24] Fernandez, R., \& Rogerson, R. (2003). Equity and resources: An analysis of education finance systems. Journal of Political Economy, $111(4), 858-897$.

[25] Evans, G. \& Tilley, J. (2011). Private schools and public divisions: The influence of private schooling on social attitudes and political choices. in Alison Park et al (eds.), British Social Attitudes: The 28th Report. London: Sage.

[26] Forman, B., \& Koch, C. (2012). Geographic Segregation: The Role of Income Inequality. Communities and Banking.

[27] Georgiadis, A., \& Manning, A. (2012). Spend it like Beckham? Inequality and redistribution in the UK, 1983-2004. Public choice, 151(34), 537-563.

[28] Golub, B., \& Jackson, M. O. (2012). How Homophily Affects the Speed of Learning and Best-Response Dynamics. The Quarterly Journal of Economics, 127(3), 1287-1338.

[29] Gouveia, M., \& Masia, N. A. (1998). Does the median voter model explain the size of government?: Evidence from the states. Public Choice, 97(1-2), 159-177. 
[30] Hoppe, H. C., Moldovanu, B., \& Sela, A. (2009). The theory of assortative matching based on costly signals. The Review of Economic Studies, 76(1), 253-281.

[31] Karadja, M., Mollerstrom, J., \& Seim, D. (2014). Richer (and Holier) than Thou? The Effect of Relative Income Improvements on Demand for Redistribution (No. 1050). George Mason University, Interdisciplinary Center for Economic Science.

[32] Kenworthy, L., \& McCall, L. (2008). Inequality, public opinion and redistribution. Socio-Economic Review, 6(1), 35-68.

[33] Kiatpongsan, S., \& Norton, M. I. (2014). How much (more) should CEOs make? A universal desire for more equal pay. Perspectives on Psychological Science, 9(6), 587-593.

[34] Kuziemko, I., Norton, M. I., Saez, E., \& Stantcheva, S. (2015). How elastic are preferences for redistribution? Evidence from randomized survey experiments. The American Economic Review, 105(4), 14781508 .

[35] Larcinese, V. (2005). Electoral competition and redistribution with rationally informed voters. Contributions in Economic Analysis \& Policy, $4(1)$.

[36] Levy, G., \& Razin, R. (2017). The Coevolution of Segregation, Polarized Beliefs, and Discrimination: The Case of Private versus State Education. American Economic Journal: Microeconomics, 9(4), 14170 .

[37] Levy, G., \& Razin, R. (2015). Preferences over equality in the presence of costly income sorting. American Economic Journal: Microeconomics, 7(2), 308-337.

[38] Matakos, K. \& Xefteris, D. (2016) Divide and Rule: Redistribution in a Model with Differentiated Candidates. forthcoming in Economic Theory

[39] Meltzer, A. H., \& Richard, S. F. (1981). A rational theory of the size of government. The Journal of Political Economy, 914-927. 
[40] Meltzer, A. H., \& Richard, S. F. (1983). Tests of a rational theory of the size of government. Public Choice, 41(3), 403-418.

[41] Milanovic, B. (2000). The median-voter hypothesis, income inequality, and income redistribution: an empirical test with the required data. European Journal of Political Economy, 16(3), 367-410.

[42] Norton, M. I., \& Ariely, D. (2011). Building a better America-One wealth quintile at a time. Perspectives on Psychological Science, 6(1), 9-12.

[43] Norton, M. I., Neal, D. T., Govan, C. L., Ariely, D., \& Holland, E. (2014). The Not-So-Common-Wealth of Australia: Evidence for a Cross-Cultural Desire for a More Equal Distribution of Wealth. Analyses of Social Issues and Public Policy, 14(1), 339-351.

[44] Pesendorfer, W. (1995). Design innovation and fashion cycles. The American Economic Review, 771-792.

[45] Piketty, T. (1995). Social mobility and redistributive politics. The Quarterly journal of economics, 551-584.

[46] Piketty, T. (2014). Capital in the Twenty-first Century. Cambridge Massachusetts: Belknap of Harvard University Press

[47] Piketty, T., Saez, E., \& Stantcheva, S. (2014). Optimal taxation of top labor incomes: A tale of three elasticities. American Economic Journal: Economic Policy, 6(1), 230-271.

[48] Rayo, L. (2013). Monopolistic Signal Provision. The BE Journal of Theoretical Economics, 13(1), 27-58.

[49] Reardon, S. F., \& Bischoff, K. (2011). Income Inequality and Income Segregation. American Journal of Sociology, 116(4), 1092-1153.

[50] Rodriguez, F. (1999). Inequality, redistribution and rent-seeking. Department of Economics, University of Maryland, 1999b.(mimeo).

[51] Rothschild, M. \& Stiglitz, J. (1970). Increasing Risk: I. A Definition. Journal of Economic Theory, 2, 225-243. 
[52] Saez, E., \& Zucman, G. (2016). Wealth Inequality in the United States since 1913: Evidence from Capitalized Income Tax Data. The Quarterly Journal of Economics, 131(2), 519-578.

[53] Savage, M. (2015). Social class in the 21st century. Penguin UK.

[54] Scervini, F. (2012). Empirics of the median voter: democracy, redistribution and the role of the middle class. The Journal of Economic Inequality, 10(4), 529-550.

[55] Veblen, T. (1899). The theory of the leisure class: An economic study in the evolution of institutions. Macmillan.

[56] Watson, T. (2009). Inequality and the measurement of residential segregation by income in American neighborhoods. Review of Income and Wealth, 55(3), 820-844.

[57] Wenglinsky, H. (2007). Are private high schools better academically than public high schools?[electronic resource]. Center on Education Policy, 2007.

[58] Windsteiger, L. (2017a). The Redistributive Consequences of Segregation.

[59] Windsteiger, L. (2017b). Sorting in the Presence of Misperceptions.

[60] Windsteiger, L. (2017c). Monopolistic Supply of Sorting, Inequality and Welfare.

\section{Appendix}

\subsection{Necessary and sufficient conditions for existence and uniqueness of a binary biased sorting equi- librium with consistency}

In this section I will derive necessary and sufficient conditions for existence and uniqueness of a binary biased sorting equilibrium with consistency in the four cases mentioned in Section 5 . 
The conditions for a binary biased sorting equilibrium can be written as

$$
\begin{aligned}
& U\left(y, \bar{E}_{p}\left(\hat{y}^{*}\right)\right)-U\left(y, \underline{E}\left(\hat{y}^{*}\right)\right)<b \forall y<\hat{y}^{*} \\
& U\left(y, \bar{E}\left(\hat{y}^{*}\right)\right)-U\left(y, \underline{E}_{r}\left(\hat{y}^{*}\right)\right) \geq b \forall y \geq \hat{y}^{*}
\end{aligned}
$$

for some $b>0$. Due to supermodularity of $U$, these two conditions can be simplified to

$$
\begin{aligned}
& U\left(\hat{y}^{*}, \bar{E}_{p}\left(\hat{y}^{*}\right)\right)-U\left(\hat{y}^{*}, \underline{E}\left(\hat{y}^{*}\right)\right) \leq b \\
& U\left(\hat{y}^{*}, \bar{E}\left(\hat{y}^{*}\right)\right)-U\left(\hat{y}^{*}, \underline{E}_{r}\left(\hat{y}^{*}\right)\right) \geq b
\end{aligned}
$$

which implies that at the equilibrium cutoff we must have

$$
U\left(\hat{y}^{*}, \bar{E}\left(\hat{y}^{*}\right)\right)-U\left(\hat{y}^{*}, \underline{E}_{r}\left(\hat{y}^{*}\right)\right) \geq U\left(\hat{y}^{*}, \bar{E}_{p}\left(\hat{y}^{*}\right)\right)-U\left(\hat{y}^{*}, \underline{E}\left(\hat{y}^{*}\right)\right)
$$

(and note that as $U\left(\hat{y}^{*}, \bar{E}\left(\hat{y}^{*}\right)\right)-U\left(\hat{y}^{*}, \underline{E}_{r}\left(\hat{y}^{*}\right)\right) \geq b$ the RHS of this inequality must be strictly positive in any biased sorting equilibrium).

Inequality (29) says that at any equilibrium cutoff it must be the case that the rich group's perceived benefit of sorting (LHS) is greater than the poor group's perceived benefit of sorting (RHS). It follows immediately that we cannot find a $b>0$ such that a biased sorting equilibrium exists at any cutoff in Case 4, in which the rich group underestimates the difference between groups for any cutoff and the poor group overestimates the difference between groups for any cutoff. The reason is that the fact that $U$ is strictly increasing in both arguments implies that the rich's perceived benefit of sorting lies below the poor's benefit of sorting for every cutoff $\hat{y}$ in this case, and therefore no positive sorting fee can be found such that the rich would be willing to pay the fee and be in the rich group and the poor would not be willing to join.

Proposition 8 No cutoff $\hat{y} \in Y$ constitutes a biased sorting equilibrium in Case 4.

Proof. The fact that in Case 4 we have $\bar{E}(\hat{y})<\bar{E}_{p}(\hat{y})$ and $\underline{E}_{r}(\hat{y})>$ $\underline{E}(\hat{y}) \forall \hat{y} \in\left(0, y_{\max }\right)$ combined with strict increasingness of $U$ implies that inequality (29) cannot be satisfied for any $\hat{y} \in Y$. 
The opposite to Case 4 happens in Case 3: if the rich group overestimates the group difference for every cutoff, while the poor group underestimates it, any cutoff $\hat{y} \in Y$ is a biased sorting equilibrium.

Proposition 9 Any cutoff $\hat{y} \in Y$ constitutes a biased sorting equilibrium in Case 3. ${ }^{14}$

Proof. Case 3 implies that $\bar{E}(\hat{y})>\bar{E}_{p}(\hat{y})$ and $\underline{E}_{r}(\hat{y})<\underline{E}(\hat{y}) \forall \hat{y} \in\left(0, y_{\max }\right)$. Together with the fact that $U$ is strictly increasing in both arguments, this implies that inequality (29) holds for all $\hat{y} \in Y$.

If we require the equilibrium partition to satisfy consistency, the equilibrium cutoff must satisfy

$$
U\left(\hat{y}^{*}, \bar{E}\left(\hat{y}^{*}\right)\right)-U\left(\hat{y}^{*}, \underline{E}_{r}\left(\hat{y}^{*}\right)\right)=U\left(\hat{y}^{*}, \bar{E}_{p}\left(\hat{y}^{*}\right)\right)-U\left(\hat{y}^{*}, \underline{E}\left(\hat{y}^{*}\right)\right)=b
$$

(and therefore both differences must be strictly positive because $b>0$ ).

Proposition 10 No cutoff $\hat{y} \in Y$ constitutes a biased sorting equilibrium with consistency in Case 3.

Proof. Case 3 implies that $\bar{E}(\hat{y})<\bar{E}_{p}(\hat{y})$ and $\underline{E}_{r}(\hat{y})>\underline{E}(\hat{y}) \forall \hat{y} \in\left(0, y_{\max }\right)$. If $U$ is strictly increasing in both arguments, condition (30) cannot be satisfied for any $\hat{y} \in Y$.

Only Case 1 and Case 2 allow for the existence of an interior biased sorting equilibrium with consistency. In fact, I find that in those two cases an interior equilibrium always exists:

Proposition 11 A biased sorting equilibrium with consistency always exists in Case 1 and Case 2.

Proof. Remember that the rich are correct at 0 and the poor at $y_{\max }$. Therefore, it holds that

$$
U(0, \bar{E}(0))-U\left(0, \underline{E}_{r}(0)\right)>(<) U\left(0, \bar{E}_{p}(0)\right)-U(0, \underline{E}(0))
$$

\footnotetext{
${ }^{14}$ If I would be meticulously diligent, I would have to exclude $\hat{y}=0$ as a potential equilibrium cutoff in this and all following Propositions. The reason is that strictly speaking $\hat{y}=0$ cannot be an equilibrium cutoff due to my definition of the partition as $\left\{[0, \hat{y}),\left[\hat{y}, y_{\max }\right]\right\} \cdot \hat{y}=0$ would imply that $S_{0}$ would be empty, which is not possible because the empty set cannot be an element of a partition. Therefore, $\hat{y}=0$ is technically not even included in my sorting equilibrium definition. If I had defined the partition the other way round, i.e. $\left\{[0, \hat{y}],\left(\hat{y}, y_{\max }\right]\right\}$, then the same would hold for $y_{\max }$.
} 
and

$U\left(y_{\max }, \bar{E}\left(y_{\max }\right)\right)-U\left(y_{\max }, \underline{E}_{r}\left(y_{\max }\right)\right)<(>) U\left(y_{\max }, \bar{E}_{p}\left(y_{\max }\right)\right)-U\left(y_{\max }, \underline{E}\left(y_{\max }\right)\right)$.

As both $U$ and the belief function are continuous, there must be an interior $\hat{y}^{*} \in\left(0, y_{\max }\right)$ such that

$$
U\left(\hat{y}^{*}, \bar{E}\left(\hat{y}^{*}\right)\right)-U\left(\hat{y}^{*}, \underline{E}_{r}\left(\hat{y}^{*}\right)\right)=U\left(\hat{y}^{*}, \bar{E}_{p}\left(\hat{y}^{*}\right)\right)-U\left(\hat{y}^{*}, \underline{E}\left(\hat{y}^{*}\right)\right) .
$$

\subsubsection{Linear utility function}

If $U$ is linear in both arguments, it is strictly speaking not in my set of analyzed utility functions, because it is not strictly increasing (and also not strictly supermodular) whenever one of the arguments is 0 . If $U(x, y)=x y$ the equilibrium condition translates to

$$
\hat{y}^{*}\left[\bar{E}-\underline{E}_{r}\right]=\hat{y}^{*}\left[\bar{E}_{p}-\underline{E}\right] .
$$

It is immediate to see that this condition will always be satisfied at $\hat{y}=0$ (also for Case 3 and Case 4). However, $\hat{y}=0$ cannot be a biased sorting equilibrium cutoff according to my definition, because I require the sorting fee $b$ to be strictly positive, and hence $U\left(\hat{y}^{*}, \bar{E}\left(\hat{y}^{*}\right)\right)-U\left(\hat{y}^{*}, \underline{E}_{r}\left(\hat{y}^{*}\right)\right)$ must be strictly positive in any equilibrium (this follows from condition (IC2)) Therefore, $\hat{y}=0$ can never constitute a biased sorting equilibrium cutoff if $U$ is linear.

For reasons of completeness, I therefore restate Propositions 8 - 10 for a linear utility function (Proposition 11 doesn't change):

Proposition 12 No cutoff $\hat{y} \in Y$ constitutes a biased sorting equilibrium in Case 4.

Proof. The fact that in Case 4 we have $\bar{E}\left(\hat{y}^{*}\right)<\bar{E}_{p}\left(\hat{y}^{*}\right)$ and $\underline{E}_{r}\left(\hat{y}^{*}\right)>$ $\underline{E}\left(\hat{y}^{*}\right) \forall \hat{y}^{*} \in\left(0, y_{\max }\right)$ combined with strict increasingness of $U$ implies that inequality (29) cannot be satisfied for any $\hat{y} \in Y$. If $U$ is linear (and hence not strictly increasing at $\hat{y}=0$ ) then inequality (29) is trivially satisfied for 
$\hat{y}=0$, but $\hat{y}=0$ cannot be a biased sorting equilibrium cutoff according to my definition, because I require the sorting fee $b$ to be strictly positive, and hence $U\left(\hat{y}^{*}, \bar{E}\left(\hat{y}^{*}\right)\right)-U\left(\hat{y}^{*}, \underline{E}_{r}\left(\hat{y}^{*}\right)\right)$ must be strictly positive in any equilibrium.

Proposition 13 Any cutoff $\hat{y} \in\left(0, y_{\max }\right]$ constitutes a biased sorting equilibrium in Case 3.

Proof. Case 3 implies that $\bar{E}\left(\hat{y}^{*}\right)>\bar{E}_{p}\left(\hat{y}^{*}\right)$ and $\underline{E}_{r}\left(\hat{y}^{*}\right)<\underline{E}\left(\hat{y}^{*}\right) \forall \hat{y}^{*} \in$ $\left(0, y_{\max }\right)$. If $U$ is strictly increasing in both arguments, this implies that inequality (29) holds for all $\hat{y} \in Y$. If $U$ is linear (and hence not strictly increasing at $\hat{y}=0$ ) then inequality (29) is trivially satisfied for $\hat{y}=0$, but $\hat{y}=0$ cannot be a biased sorting equilibrium cutoff.

Proposition 14 No cutoff $\hat{y} \in\left(0, y_{\max }\right)$ constitutes a biased sorting equilibrium with consistency in Case 3.

Proof. Case 3 implies that $\bar{E}\left(\hat{y}^{*}\right)<\bar{E}_{p}\left(\hat{y}^{*}\right)$ and $\underline{E}_{r}\left(\hat{y}^{*}\right)>\underline{E}\left(\hat{y}^{*}\right) \forall \hat{y}^{*} \in$ $\left(0, y_{\max }\right)$. If $U$ is strictly increasing everywhere, condition (30) cannot be satisfied for any $\hat{y} \in Y$. If $U$ is linear then the first equality of condition (30) is trivially satisfied for $\hat{y}=0$, but the second equality can never be satisfied, because $b>0$..

\subsubsection{Uniqueness: Linear utility function}

For Case 1 and Case 2, the following sufficient conditions for uniqueness of an interior biased sorting equilibrium with consistency can be stated if $U$ is linear:

Proposition 15 If people are biased according to Case 1 or Case $2, U$ is linear in both arguments and people's misperceptions converge to the truth monotonically, i.e.

$$
\frac{d\left|\bar{E}(\hat{y})-\bar{E}_{p}(\hat{y})\right|}{d \hat{y}}<0 \quad \text { and } \quad \frac{d\left|\underline{E}_{r}(\hat{y})-\underline{E}(\hat{y})\right|}{d \hat{y}}>0 \quad \forall \hat{y} \in\left(0, y_{\max }\right)
$$

there always exists a unique biased sorting equilibrium with consistency. 
Proof. The equilibrium cutoff must satisfy

$$
\bar{E}\left(\hat{y}^{*}\right)-\underline{E}_{r}\left(\hat{y}^{*}\right)=\bar{E}_{p}\left(\hat{y}^{*}\right)-\underline{E}\left(\hat{y}^{*}\right) .
$$

Suppose people are biased according to Case 1 (the argument can be made analogously for Case 2). Then the conditions in (31) become

$$
\frac{d\left(\bar{E}(\hat{y})-\bar{E}_{p}(\hat{y})\right)}{d \hat{y}}<0 \quad \text { and } \quad \frac{d\left(\underline{E}_{r}(\hat{y})-\underline{E}(\hat{y})\right)}{d \hat{y}}>0 \quad \forall \hat{y} \in\left(0, y_{\max }\right)
$$

This implies that the distance between the correct difference in average group incomes, $\underline{E}(\hat{y})-\underline{E}(\hat{y})$, and the poor's perceived group difference, $\bar{E}_{p}(\hat{y})-\underline{E}(\hat{y})$, which can be written as $\bar{E}(\hat{y})-\bar{E}_{p}(\hat{y})$, is monotonically decreasing in $\hat{y}$, while the opposite holds for the distance between the correct group difference and the rich's perceived group difference (which can be written as $\left.\underline{E}_{r}(\hat{y})-\underline{E}(\hat{y})\right)$. This means that there can be only one $\hat{y}$ for which the distance between the correct group differences and the group's perceived group differences is the same, and therefore (31) guarantees that the perceived benefits of sorting of the rich and of the poor only cut once in the interval $\left(0, y_{\max }\right)$.

\subsubsection{Uniqueness in Case 1: General utility function}

For a general utility function, we also need to impose conditions on the shape of the utility function to ensure uniqueness.

Proposition 16 If people are biased according to Case 1 and people's misperceptions converge to the truth monotonically, i.e.

$$
\frac{d\left|\bar{E}(\hat{y})-\bar{E}_{p}(\hat{y})\right|}{d \hat{y}}<0 \quad \text { and } \quad \frac{d\left|\underline{E}_{r}(\hat{y})-\underline{E}(\hat{y})\right|}{d \hat{y}}>0 \quad \forall \hat{y} \in\left(0, y_{\max }\right)
$$

and additionally it holds that at any $\hat{y}^{*}$ for which

$$
U\left(\hat{y}^{*}, \bar{E}\left(\hat{y}^{*}\right)\right)-U\left(\hat{y}^{*}, \underline{E}_{r}\left(\hat{y}^{*}\right)\right)=U\left(\hat{y}^{*}, \bar{E}_{p}\left(\hat{y}^{*}\right)\right)-U\left(\hat{y}^{*}, \underline{E}\left(\hat{y}^{*}\right)\right)
$$

holds we have that

$$
U_{1}\left(\hat{y}^{*}, \bar{E}\left(\hat{y}^{*}\right)\right)-U_{1}\left(\hat{y}^{*}, \underline{E}_{r}\left(\hat{y}^{*}\right)\right) \leq U_{1}\left(\hat{y}^{*}, \bar{E}_{p}\left(\hat{y}^{*}\right)\right)-U_{1}\left(\hat{y}^{*}, \underline{E}\left(\hat{y}^{*}\right)\right)
$$




$$
U_{2}\left(\hat{y}^{*}, \underline{E}_{r}\left(\hat{y}^{*}\right)\right) \geq U_{2}\left(\hat{y}^{*}, \underline{E}\left(\hat{y}^{*}\right)\right)
$$

and

$$
U_{2}\left(\hat{y}^{*}, \bar{E}\left(\hat{y}^{*}\right)\right) \leq U_{2}\left(\hat{y}^{*}, \bar{E}_{p}\left(\hat{y}^{*}\right)\right)
$$

there always exists a unique biased sorting equilibrium with consistency.

Proof. At any equilibrium cutoff $\hat{y}^{*}$ such that

$$
U\left(\hat{y}^{*}, \bar{E}\left(\hat{y}^{*}\right)\right)-U\left(\hat{y}^{*}, \underline{E}_{r}\left(\hat{y}^{*}\right)\right)=U\left(\hat{y}^{*}, \bar{E}_{p}\left(\hat{y}^{*}\right)\right)-U\left(\hat{y}^{*}, \underline{E}\left(\hat{y}^{*}\right)\right)
$$

these conditions ensure that the slope of the function on the LHS is smaller than the slope of the function on the RHS, which implies that the two functions can only intersect once.

Remark 2 The conditions in Proposition 16 are sufficient conditions for uniqueness, because they ensure that the slope of the left hand side of the equilibrium condition is strictly smaller than the slope of the right hand side at any intersection.

Remark 3 Similar sufficient conditions can be found for Case 2 type misperceptions, where both groups overestimate group differences.

\subsection{Biased sorting equilibria with more than two groups: General proofs}

\subsubsection{Proof that with the uniform distribution, arbitrarily many groups are possible with Proportional Biased Beliefs}

Suppose people have proportional biased beliefs of type 1. If a partition $\left[0, \hat{y}_{1}, \ldots, \hat{y}_{n-1}, y_{\max }\right]$ is a biased sorting equilibrium with local consistency, it needs to satisfy

$$
E^{i}-E_{i}^{i-1}=E_{i-1}^{i}-E^{i-1} \quad \forall i
$$

and

$$
y E^{i}(P)-y E_{i}^{k}(P) \geq b_{i}-b_{k} \quad \forall y \in S_{b_{i}}, \forall i, \forall k \neq i .
$$


Given the functional form of the misperceptions, (32) can be written as

$$
\left(\hat{y}^{i}-E^{i-1}\right)\left(1-F_{i+1}+F_{i}\right)=\left(1-F_{i}+F_{i-1}\right)\left(E^{i}-\hat{y}_{i}\right)
$$

If the income distribution is uniform on $[0, a]$, this expression becomes

$$
\frac{\left(a-\hat{y}_{i+1}+\hat{y}_{i}\right)}{a}\left(\frac{\hat{y}_{i}-\hat{y}_{i-1}}{2}\right)=\frac{\left(a-\hat{y}_{i}+\hat{y}_{i-1}\right)}{a}\left(\frac{\hat{y}_{i+1}-\hat{y}_{i}}{2}\right) \quad \forall i
$$

which, after simplifying, yields

$$
\hat{y}_{i}=\frac{\hat{y}_{i+1}+\hat{y}_{i-1}}{2} \quad \forall i
$$

and therefore that all cutoffs need to be equidistant in equilibrium. If all cutoffs are equidistant, the cutoffs will all be of the form

$$
y_{i}=\frac{i a}{n}
$$

(if there's $n$ groups in total). Also, because all groups are of equal size (in terms of $F$ ), their misperceptions about the other groups are equally severe. Hence, all groups below a given group $i$ have equal (wrong) beliefs about average income in the groups above group $i$ and equally, all groups above group $i$ have equal (wrong) beliefs about the groups below group $i$ (because the severity of the misperceptions depends on group size and all groups are of equal size (in terms of $F$ ) in this case). Therefore, in order to check whether condition (33) holds, it suffices to look at one group (e.g. group 0) and check whether everybody in this group prefers staying where they are to switching to any other group with higher average income, and vice versa whether everybody in those groups prefers staying to switching to group 0 . If group 0 wants to stay where they are and no other groups want to switch to group 0 , then this is also satisfied for any other group $i$, because what matters for this decision is not the group's location on the income line itself, but rather the distance (in terms of $F$ ) to the other groups. As cutoffs are equidistant, all groups consider the same range of distances to each other when they decide whether they want to switch or not, so it suffices to consider one "model" group's decisions.

With the uniform distribution and equidistant cutoffs, it turns out that 
all $b_{i} \mathrm{~s}$ are of the form

$$
b_{i}=\left(\frac{a}{n}\right)^{2}\left(1-\beta \frac{n-1}{2 n}\right) \frac{i(i+1)}{2}
$$

Furthermore, group $i$ 's belief about average income in another group $j$ is

$$
E_{i}^{j}=E^{j}+\beta \frac{n-1}{n} \frac{a}{2 n}
$$

if $i>j$ and

$$
E_{i}^{j}=E^{j}-\beta \frac{n-1}{n} \frac{a}{2 n}
$$

if $i<j$.

If group 0 doesn't want to switch to group 2, it has to hold that

$$
\hat{y}_{1}\left(E_{0}^{2}-E^{0}\right) \leq b_{2}
$$

which translates to

$$
\left(\frac{a}{n}\right)^{2}\left(2-\beta \frac{n-1}{2 n}\right) \leq 3\left(1-\beta \frac{n-1}{2 n}\right)\left(\frac{a}{n}\right)^{2}
$$

and therefore

$$
\beta \frac{n-1}{n} \leq 1
$$

which is always satisfied because $\beta \in(0,1)$. As only group distances matter in this case, condition (34) ensures that any group doesn't want to switch to a group that is two cutoffs above their own (i.e. if condition (34) is satisfied, then group 1 doesn't want to switch to group 3, group 2 doesn't want to switch to group 4 and so on).

If group 2 doesn't want to switch to group 0 it has to hold that

$$
\hat{y}_{2}\left(E^{2}-E_{2}^{0}\right) \geq b_{2}
$$

which translates to

$$
2\left(\frac{a}{n}\right)^{2}\left(2-\beta \frac{n-1}{2 n}\right) \geq 3\left(1-\beta \frac{n-1}{2 n}\right)\left(\frac{a}{n}\right)^{2}
$$


and therefore

$$
\beta \frac{n-1}{2 n} \geq-1
$$

which always holds. Again, this condition also ensures that group 3 doesn't want to switch to group 1, group 4 to group 2 etc. In a similar way we can check conditions for larger distances (group 0 to group 3, etc.). It turns out that these conditions are even slacker than the ones above, and therefore that all conditions of the form (33) are satisfied if $F$ is uniform and the cutoffs are equidistant.

It is immediate to see that the proof works in the analogous way if people have proportional biased beliefs of type 2 .

Proposition 17 If people have proportional biased beliefs, and the income distribution is uniform, any equidistant partition is a biased sorting equilibrium with local consistency.

\subsubsection{General income distribution, local consistency}

Concerning general income distributions, I will first analyze the case of three groups. I will assume that people have proportional biased beliefs of type 1 (Everything can be done analogously for proportional biased beliefs of type 2):

$$
\begin{aligned}
& E_{0}^{0}=E^{0} \\
& E_{0}^{1}=\beta\left(1-F\left(\hat{y}_{1}\right)\right) \hat{y}_{1}+\left(1-\beta\left(1-F\left(\hat{y}_{1}\right)\right)\right) E^{1} \\
& E_{0}^{2}=\beta\left(1-F\left(\hat{y}_{1}\right)\right) \hat{y}_{2}+\left(1-\beta\left(1-F\left(\hat{y}_{1}\right)\right)\right) E^{2} \\
& E_{1}^{0}=\beta\left(1-F\left(\hat{y}_{2}\right)+F\left(\hat{y}_{1}\right)\right) \hat{y}_{1}+\left(1-\beta\left(1-F\left(\hat{y}_{2}\right)+F\left(\hat{y}_{1}\right)\right)\right) E^{0} \\
& E_{1}^{1}=E^{1} \\
& E_{1}^{2}=\beta\left(1-F\left(\hat{y}_{2}\right)+F\left(\hat{y}_{1}\right)\right) \hat{y}_{2}+\left(1-\beta\left(1-F\left(\hat{y}_{2}\right)+F\left(\hat{y}_{1}\right)\right)\right) E^{2} \\
& E_{2}^{0}=\beta F\left(\hat{y}_{2}\right) \hat{y}_{1}+\left(1-\beta F\left(\hat{y}_{2}\right)\right) E^{0} \\
& E_{2}^{1}=\beta F\left(\hat{y}_{2}\right) \hat{y}_{2}+\left(1-\beta F\left(\hat{y}_{2}\right)\right) E^{1} \\
& E_{2}^{2}=E^{2}
\end{aligned}
$$


A biased sorting equilibrium with local consistency and three groups needs to satisfy the following equilibrium conditions

$$
\begin{aligned}
& E_{0}^{1}-E_{0}^{0}=E_{1}^{1}-E_{1}^{0} \\
& E_{1}^{2}-E_{1}^{1}=E_{2}^{2}-E_{2}^{1}
\end{aligned}
$$

and

$$
\begin{aligned}
& \hat{y}_{1}\left(E_{0}^{2}-E^{0}\right) \leq b_{2} \\
& \hat{y}_{2}\left(E^{2}-E_{2}^{0}\right) \geq b_{2}
\end{aligned}
$$

Given the functional form of the misperceptions defined above, conditions (35) and (36) can be rewritten as

$$
\begin{gathered}
G\left(\hat{y}_{1}, \hat{y}_{2}\right)=\frac{\left(1-F_{2}+F_{1}\right) E^{0}+\left(1-F_{1}\right) E^{1}}{2-F_{2}}-\hat{y}_{1}=0 \\
H\left(\hat{y}_{1}, \hat{y}_{2}\right)=\frac{F_{2} E^{1}+\left(1-F_{2}+F_{1}\right) E^{2}}{1+F_{1}}-\hat{y}_{2}=0
\end{gathered}
$$

An equilibrium will exist if there exists a pair $\left(\hat{y}_{1}^{*}, \hat{y}_{2}^{*}\right)$ that satisfy both (39) and (40). My proof for existence proceeds in the following way: first I show that $(0,0)$ and $\left(E, y_{\max }\right)$ satisfy $(39)$, then I show that $\left(y_{\max }, y_{\max }\right)$ and $(0, E)$ satisfy (40). This implies that, if $G$ is such that $G\left(\hat{y}_{1}, \hat{y}_{2}\right)=0$ implicitly describes a continuous function $\hat{y}_{2}\left(\hat{y}_{1}\right)$ and $H$ is such that $H\left(\hat{y}_{1}, \hat{y}_{2}\right)=0$ implicitly describes a continuous function $\hat{y}_{1}\left(\hat{y}_{2}\right)$, then the two must cross at some point in the $\left(\hat{y}_{1}, \hat{y}_{2}\right)$ space (and in fact this crossing must be where $\hat{y}_{1} \leq E$ and $\hat{y}_{2} \geq E$ ). I then invoke the implicit function theorem to show that these two continuous functions exist.

Step 1: $(0,0)$ and $\left(E, y_{\max }\right)$ satisfy $(39)$ :

If I set $\hat{y}_{1}$ to 0 it follows that $F_{1}=0$ and $E^{0}=0$ and therefore

$$
G\left(0, \hat{y}_{2}\right)=\frac{E^{1}}{1-F_{2}}-0
$$

and hence (39) is satisfied if $\hat{y}_{2}=0$ (which implies that $E^{1}=0$ and therefore $G(0,0)=0)$. 
If I set $\hat{y}_{2}$ to $y_{\max }$ it follows that $F_{2}=1$ and therefore

$$
G\left(\hat{y}_{1}, y_{\max }\right)=F_{1} E^{0}+\left(1-F_{1}\right) E^{1}-\hat{y}_{1}
$$

and hence (39) is satisfied if $\hat{y}_{1}=E$.

Step 2: $\left(y_{\max }, y_{\max }\right)$ and $(0, E)$ satisfy $(40)$ :

If I set $\hat{y}_{2}$ to $y_{\max }$ then $F_{2}=1$ and $E^{2}=y_{\max }$ and therefore

$$
H\left(\hat{y}_{1}, y_{\max }\right)=\frac{E^{1}+F_{1} y_{\max }}{1+F_{1}}-y_{\max }
$$

and hence (40) is satisfied if $\hat{y}_{1}=y_{\max }$ (because then $E^{1}=y_{\max }$ and $F_{1}=1$ and hence $\left.H\left(y_{\max }, y_{\max }\right)=\frac{2 y_{\max }}{2}-y_{\max }=0\right)$.

If $I$ set $\hat{y}_{1}$ to 0 it follows that $F_{1}=0$ and therefore

$$
H\left(0, \hat{y}_{2}\right)=F_{2} E^{1}+\left(1-F_{2}\right) E^{2}-\hat{y}_{2}
$$

and hence (40) is satisfied if $\hat{y}_{2}=E$.

Step 3: $G\left(\hat{y}_{1}, \hat{y}_{2}\right)=0$ describes a continuous function $\hat{y}_{2}\left(\hat{y}_{1}\right)$ for all $\left(\hat{y}_{1}, \hat{y}_{2}\right) \neq(0,0)$ satisfying $G\left(\hat{y}_{1}, \hat{y}_{2}\right)=0$ :

Note that ${ }^{15}$

$$
\begin{aligned}
\frac{\partial G}{\partial \hat{y}_{2}}= & \frac{\left[-f_{2} E^{0}+\left(1-F_{1}\right) \frac{\partial E^{1}}{\partial \hat{y}_{2}}\right]\left(2-F_{2}\right)+\left[\left(1-F_{2}+F_{1}\right) E^{0}+\left(1-F_{1}\right) E^{1}\right] f_{2}}{\left(2-F_{2}\right)^{2}} \\
& =\frac{f_{2}}{\left(2-F_{2}\right)^{2}}\left[\left(1-F_{1}\right)\left(E^{1}-E^{0}\right)+\frac{\left(1-F_{1}\right)\left(2-F_{2}\right)\left(\hat{y}_{2}-E^{1}\right)}{F_{2}-F_{1}}\right]
\end{aligned}
$$

By the implicit function theorem, $G\left(\hat{y}_{1}, \hat{y}_{2}\right)=0$ describes a continuous function $\hat{y}_{2}\left(\hat{y}_{1}\right)$ for all $\left(\hat{y}_{1}, \hat{y}_{2}\right)$ satisfying $G\left(\hat{y}_{1}, \hat{y}_{2}\right)=0$ such that

$$
\frac{\partial G\left(\hat{y}_{1}, \hat{y}_{2}\right)}{\partial \hat{y}_{2}} \neq 0
$$

If $f_{2} \neq 0$ (I assume that $F$ is such that this holds whenever $\hat{y}_{2} \neq 0$ ) then

$$
\frac{\partial G}{\partial \hat{y}_{2}} \neq 0 \Longleftrightarrow\left(1-F_{1}\right)\left(E^{1}-E^{0}\right)+\frac{\left(1-F_{1}\right)\left(2-F_{2}\right)\left(\hat{y}_{2}-E^{1}\right)}{F_{2}-F_{1}} \neq 0
$$

\footnotetext{
${ }^{15}$ Notation: $f_{i}=f\left(\hat{y}_{i}\right)$
} 
In fact, it is easy to see that the latter inequality would not hold if

$$
1-F_{1}=0
$$

but this cannot happen, because it would imply that $\hat{y}_{1}=y_{\max }$ and $G\left(y_{\max }, \hat{y}_{2}\right)=$ 0 is not satisfied for any $\hat{y}_{2}$. Furthermore, this expression is zero if

$$
E^{1}=E^{0} \text { and } \hat{y}_{2}=E^{1}
$$

The only point at which this would be satisfied is where $\left(\hat{y}_{1}, \hat{y}_{2}\right)=(0,0)$. On all points $\left(\hat{y}_{1}, \hat{y}_{2}\right) \neq(0,0)$ for which $G\left(\hat{y}_{1}, \hat{y}_{2}\right)=0$ it is actually the case that

$$
\frac{\partial G\left(\hat{y}_{1}, \hat{y}_{2}\right)}{\partial \hat{y}_{2}}>0
$$

because $E^{2}-E^{1}>0$ and $\hat{y}_{2}-E^{2}>0$.

Step 4: $H\left(\hat{y}_{1}, \hat{y}_{2}\right)=0$ describes a continuous function $\hat{y}_{1}\left(\hat{y}_{2}\right)$ for all points $\left(\hat{y}_{1}, \hat{y}_{2}\right) \neq\left(y_{\max }, y_{\max }\right)$ and $\left(\hat{y}_{1}, \hat{y}_{2}\right) \neq(0, E)$ for which $H\left(\hat{y}_{1}, \hat{y}_{2}\right)=0$ :

$$
\begin{aligned}
\frac{\partial H}{\partial \hat{y}_{1}} & =\frac{\left.\left[f_{1} E^{2}+F_{2} \frac{\partial E^{1}}{\partial \hat{y}_{1}}\right]\left(1+F_{1}\right)-F_{2} E^{1}+\left(1-F_{2}+F_{1}\right) E^{2}\right] f_{1}}{\left(1+F_{1}\right)^{2}} \\
& =\frac{f_{1}}{\left(1+F_{1}\right)^{2}}\left[F_{2}\left(E^{2}-E^{1}\right)+F_{2} \frac{\left(1+F_{1}\right)\left(E^{1}-\hat{y}_{1}\right)}{F_{2}-F_{1}}\right]
\end{aligned}
$$

By the implicit function theorem, $H\left(\hat{y}_{1}, \hat{y}_{2}\right)=0$ describes a continuous function $\hat{y}_{1}\left(\hat{y}_{2}\right)$ for all $\left(\hat{y}_{1}, \hat{y}_{2}\right)$ satisfying $H\left(\hat{y}_{1}, \hat{y}_{2}\right)=0$ such that

$$
\frac{\partial H\left(\hat{y}_{1}, \hat{y}_{2}\right)}{\partial \hat{y}_{1}} \neq 0
$$

If $f_{1} \neq 0$ (I assume that $F$ is such that this holds whenever $\hat{y}_{1} \neq 0$ ) then

$$
\frac{\partial H}{\partial \hat{y}_{1}} \neq 0 \Longleftrightarrow F_{2}\left(E^{2}-E^{1}\right)+F_{2} \frac{\left(1+F_{1}\right)\left(E^{1}-\hat{y}_{1}\right)}{F_{2}-F_{1}} \neq 0
$$

In fact, it is easy to see that the latter inequality would not hold if

$$
F_{2}=0
$$

which implies that in order for $\frac{\partial H}{\partial \hat{y}_{1}} \neq 0$ we need $\hat{y}_{2} \neq 0$. However, note 
that $\hat{y}_{2}$ will never be 0 along $H\left(\hat{y}_{1}, \hat{y}_{2}\right)=0$ because $H\left(\hat{y}_{1}, 0\right)=0$ is never satisfied. Furthermore, the expression is zero if

$$
E^{2}=E^{1} \text { and } E^{1}=\hat{y}_{1}
$$

The only point at which this would be satisfied is at $\left(y_{\max }, y_{\max }\right)$. On all points $\left(\hat{y}_{1}, \hat{y}_{2}\right) \neq\left(y_{\max }, y_{\max }\right)$ for which $H\left(\hat{y}_{1}, \hat{y}_{2}\right)=0$ it is actually the case that

$$
\frac{\partial H}{\partial \hat{y}_{1}}>0
$$

because $E^{2}-E^{1}>0$ and $E^{1}-\hat{y}_{1}>0$.

The above steps establish that there exists a pair $\left(\hat{y}_{1}, \hat{y}_{2}\right)$ that satisfy (39) and (40). Whether they also satisfy (37) and (38) and therefore constitute a biased sorting equilibrium with local consistency depends on $\beta$ : We know that

$$
b_{2}=\hat{y}_{2}\left(E^{2}-E_{2}^{1}\right)+b_{1}=\hat{y}_{2}\left(E_{1}^{2}-E^{1}\right)+b_{1}
$$

and

$$
b_{1}=\hat{y}_{1}\left(E^{1}-E_{1}^{0}\right)=\hat{y}_{1}\left(E_{0}^{1}-E^{0}\right)
$$

Note that (37) and (41) can be combined to give

$$
\hat{y}_{1}\left(E_{0}^{2}-E_{0}^{1}\right) \leq \hat{y}_{2}\left(E_{1}^{2}-E^{1}\right)
$$

(rewrite $\hat{y}_{1}\left(E_{0}^{2}-E^{0}\right)=\hat{y}_{1}\left(E_{0}^{2}-E_{0}^{1}+E_{0}^{1}-E^{0}\right)$ and note that $\hat{y}_{1}\left(E_{0}^{1}-E^{0}\right)=b_{1}$ and $(36)$ can be written as $\left.\hat{y}_{2}\left(E_{1}^{2}-E_{1}^{1}\right)=\hat{y}_{2}\left(E_{2}^{2}-E_{2}^{1}\right)=b_{2}-b_{1}\right)$. Denoting by $1-x$ and $1-w$ the size of group 0 and group 1 respectively, (42) can be rearranged to give

$\hat{y}_{1}\left(E^{2}-E^{1}\right)+\beta\left(x\left(E^{1}-\hat{y}_{1}-E^{2}+\hat{y}_{2}\right) \hat{y}_{1}+w\left(E^{2}-\hat{y}_{2}\right) \hat{y}_{2}\right) \leq \hat{y}_{2}\left(E^{2}-E^{1}\right)$.

As

$$
\hat{y}_{1}\left(E^{2}-E^{1}\right)<\hat{y}_{2}\left(E^{2}-E^{1}\right),
$$

condition (42) always holds if $\beta$ is small enough. We can deal with (38) in 
the analogous way: Rewriting it, we get

$$
\hat{y}_{2}\left(E^{2}-E_{2}^{1}\right)+\hat{y}_{2}\left(E_{2}^{1}-E_{2}^{0}\right) \geq b_{2}
$$

and because (41) yields $\hat{y}_{2}\left(E^{2}-E_{2}^{1}\right)=b_{2}-b_{1}$ we get $\hat{y}_{2}\left(E_{2}^{1}-E_{2}^{0}\right) \geq b_{1}$ which is equivalent to

$$
\hat{y}_{2}\left(E_{2}^{1}-E_{2}^{0}\right) \geq \hat{y}_{1}\left(E_{0}^{1}-E^{0}\right) .
$$

Denoting by $1-x$ and $1-z$ the size of group 0 and 2 respectively, this condition can be rearranged to give

$$
\hat{y}_{2}\left(E^{1}-E^{0}\right)+\beta\left(z\left(\hat{y}_{2}-E^{1}-\hat{y}_{1}+E^{0}\right) \hat{y}_{2}+x\left(E^{1}-\hat{y}_{1}\right) \hat{y}_{1}\right) \geq \hat{y}_{1}\left(E^{1}-E^{0}\right) .
$$

Again, due to

$$
\hat{y}_{2}\left(E^{1}-E^{0}\right)>\hat{y}_{1}\left(E^{1}-E^{0}\right)
$$

condition (43) always holds for small enough $\beta$. Hence, conditions (37) and (37) will always be satisfied for small $\beta$. It follows that for small enough $\beta$ there will always exist a pair $\left(\hat{y}_{1}, \hat{y}_{2}\right)$ that satisfy all four conditions $(39)$ (38).

Proposition 18 With Proportional Biased Beliefs, a biased sorting equilibrium with local consistency and three groups will always exist for small enough $\beta$.

In fact, it can be seen that the same pattern emerges for four groups:

With four groups, the conditions for equilibrium and local consistency can be reduced to

$$
\begin{aligned}
& E_{0}^{1}-E^{0}=E^{1}-E_{1}^{0} \\
& E_{1}^{2}-E^{1}=E^{2}-E_{2}^{1} \\
& E_{2}^{3}-E^{2}=E^{3}-E_{3}^{2}
\end{aligned}
$$


and

$$
\begin{aligned}
& \hat{y}_{1}\left(E_{0}^{2}-E^{0}\right) \leq b_{2} \\
& \hat{y}_{2}\left(E^{2}-E_{2}^{0}\right) \geq b_{2} \\
& \hat{y}_{1}\left(E_{0}^{3}-E^{0}\right) \leq b_{3} \\
& \hat{y}_{3}\left(E^{3}-E_{3}^{0}\right) \geq b_{3} \\
& \hat{y}_{2}\left(E_{1}^{3}-E^{1}\right) \leq b_{3}-b_{1} \\
& \hat{y}_{3}\left(E^{3}-E_{3}^{1}\right) \geq b_{3}-b_{1}
\end{aligned}
$$

The first three conditions yield

$$
\begin{gathered}
\hat{y}_{1}=\frac{\left(1-F_{2}+F_{1}\right) E^{0}+\left(1-F_{1}\right) E^{1}}{2-F_{2}} \\
\hat{y}_{2}=\frac{\left(1-F_{3}+F_{2}\right) E^{1}+\left(1-F_{2}+F_{1}\right) E^{2}}{2+F_{1}-F_{3}} \\
\hat{y}_{3}=\frac{\left(1-F_{3}+F_{2}\right) E^{3}+\left(F_{3}\right) E^{2}}{1+F_{2}}
\end{gathered}
$$

Again, the equilibrium cutoffs are weighted averages of the average incomes of the two neighbouring groups and such cutoffs can always be found. The additional six conditions (which ensure that people don't want to switch to another non-neighbouring group) are again satisfied if $\beta$ is small enough (the proof proceeds in the same way as for the three-group case above). This pattern of equilibrium cutoffs that are weighted averages of neighbouring groups' average incomes emerges for every finite number of groups. Thus, if only local consistency is required, existence of multi-group biased sorting equilibria is guaranteed for small $\beta$. As I demonstrate above, if the income distribution is uniform, any equidistant partition is a sorting equilibrium, irrespective of the size of $\beta$.

\subsubsection{General income distribution, global consistency}

In Section 6, I have pointed out that for the existence of biased sorting equilibria with global consistency and more than two groups, it is crucial how exactly the misperceptions are modelled and I have demonstrated that equilibria with more than two groups do not exist under the assumption 
that perceived differences between groups decline with group distance. In the following, I will show that if people have proportional biased beliefs there cannot exist more than three groups in equilibrium.

Suppose there are four groups: A poor group with income from 0 to $\hat{y}_{1}$, a lower middle-class group with incomes from $\hat{y}_{1}$ to $\hat{y}_{2}$, an upper middleclass group with incomes from $\hat{y}_{2}$ to $\hat{y}_{3}$ and a rich group with incomes from $\hat{y}_{3}$ to $y_{\max }$. As always, I assume that people perceive the average income in their own group correctly, but they are biased about average income in the other groups. Suppose that people have proportional biased beliefs of type $1:^{16}$

$$
\begin{aligned}
& E_{0}^{0}=E^{0} \\
& E_{0}^{1}=\beta\left(1-F\left(\hat{y}_{1}\right)\right) \hat{y}_{1}+\left(1-\beta\left(1-F\left(\hat{y}_{1}\right)\right)\right) E^{1} \\
& E_{0}^{2}=\beta\left(1-F\left(\hat{y}_{1}\right)\right) \hat{y}_{2}+\left(1-\beta\left(1-F\left(\hat{y}_{1}\right)\right)\right) E^{2} \\
& E_{0}^{3}=\beta\left(1-F\left(\hat{y}_{1}\right)\right) \hat{y}_{3}+\left(1-\beta\left(1-F\left(\hat{y}_{1}\right)\right)\right) E^{3} \\
& E_{1}^{0}=\beta\left(1-F\left(\hat{y}_{2}\right)+F\left(\hat{y}_{1}\right)\right) \hat{y}_{1}+\left(1-\beta\left(1-F\left(\hat{y}_{2}\right)+F\left(\hat{y}_{1}\right)\right)\right) E^{0} \\
& E_{1}^{1}=E^{1} \\
& E_{1}^{2}=\beta\left(1-F\left(\hat{y}_{2}\right)+F\left(\hat{y}_{1}\right)\right) \hat{y}_{2}+\left(1-\beta\left(1-F\left(\hat{y}_{2}\right)+F\left(\hat{y}_{1}\right)\right)\right) E^{2} \\
& E_{1}^{3}=\beta\left(1-F\left(\hat{y}_{2}\right)+F\left(\hat{y}_{1}\right)\right) \hat{y}_{3}+\left(1-\beta\left(1-F\left(\hat{y}_{2}\right)+F\left(\hat{y}_{1}\right)\right)\right) E^{3} \\
& E_{2}^{0}=\beta\left(1-F\left(\hat{y}_{3}\right)+F\left(\hat{y}_{2}\right)\right) \hat{y}_{1}+\left(1-\beta\left(1-F\left(\hat{y}_{3}\right)+F\left(\hat{y}_{2}\right)\right)\right) E^{0} \\
& E_{2}^{1}=\beta\left(1-F\left(\hat{y}_{3}\right)+F\left(\hat{y}_{2}\right)\right) \hat{y}_{2}+\left(1-\beta\left(1-F\left(\hat{y}_{3}\right)+F\left(\hat{y}_{2}\right)\right)\right) E^{1} \\
& E_{2}^{2}=E^{2} \\
& E_{2}^{3}=\beta\left(1-F\left(\hat{y}_{3}\right)+F\left(\hat{y}_{2}\right)\right) \hat{y}_{3}+\left(1-\beta\left(1-F\left(\hat{y}_{3}\right)+F\left(\hat{y}_{2}\right)\right)\right) E^{3} \\
& E_{3}^{0}=\beta F\left(\hat{y}_{3}\right) \hat{y}_{1}+\left(1-\beta F\left(\hat{y}_{3}\right)\right) E^{0} \\
& E_{3}^{1}=\beta F\left(\hat{y}_{3}\right) \hat{y}_{2}+\left(1-\beta F\left(\hat{y}_{3}\right)\right) E^{1} \\
& E_{3}^{2}=\beta F\left(\hat{y}_{3}\right) \hat{y}_{3}+\left(1-\beta F\left(\hat{y}_{3}\right)\right) E^{2} \\
& E_{3}^{3}=E^{3}
\end{aligned}
$$

Then in order for this partition to constitute a biased sorting equilibrium with global consistency, the following conditions have to hold:

For a biased sorting equilibrium, I need:

\footnotetext{
${ }^{16}$ The analysis for proportional biased beliefs of type 2 can be done in the analogous way.
} 


$$
\begin{aligned}
& y\left(E_{0}^{1}-E_{0}^{0}\right) \leq b_{1} \quad \forall y \leq \hat{y}_{1} \\
& y\left(E_{0}^{2}-E_{0}^{0}\right) \leq b_{2} \quad \forall y \leq \hat{y}_{1} \\
& y\left(E_{0}^{2}-E_{0}^{0}\right) \leq b_{3} \quad \forall y \leq \hat{y}_{1} \\
& y\left(E_{1}^{1}-E_{1}^{0}\right) \geq b_{1} \quad \forall y \in\left[\hat{y}_{1}, \hat{y}_{2}\right] \\
& y\left(E_{1}^{2}-E_{1}^{1}\right) \leq b_{2}-b_{1} \quad \forall y \in\left[\hat{y}_{1}, \hat{y}_{2}\right] \\
& y\left(E_{1}^{3}-E_{1}^{1}\right) \leq b_{3}-b_{1} \quad \forall y \in\left[\hat{y}_{1}, \hat{y}_{2}\right] \\
& y\left(E_{2}^{2}-E_{2}^{0}\right) \geq b_{2} \quad \forall y \in\left[\hat{y}_{2}, \hat{y}_{3}\right] \\
& y\left(E_{2}^{2}-E_{2}^{1}\right) \geq b_{2}-b_{1} \quad \forall y \in\left[\hat{y}_{2}, \hat{y}_{3}\right] \\
& y\left(E_{2}^{3}-E_{2}^{2}\right) \leq b_{3}-b_{2} \quad \forall y \in\left[\hat{y}_{2}, \hat{y}_{3}\right] \\
& y\left(E_{3}^{3}-E_{3}^{0}\right) \geq b_{3} \quad \forall y \geq \hat{y}_{3} \\
& y\left(E_{3}^{3}-E_{3}^{1}\right) \geq b_{3}-b_{1} \quad \forall y \geq \hat{y}_{3} \\
& y\left(E_{3}^{3}-E_{3}^{2}\right) \geq b_{3}-b_{2} \quad \forall y \geq \hat{y}_{3}
\end{aligned}
$$

For global consistency, the additional conditions are:

$$
\begin{aligned}
& y\left(E_{0}^{1}-E_{0}^{0}\right) \geq b_{1} \quad \forall y \in\left[\hat{y}_{1}, \hat{y}_{2}\right] \\
& y\left(E_{0}^{2}-E_{0}^{1}\right) \leq b_{2}-b_{1} \quad \forall y \in\left[\hat{y}_{1}, \hat{y}_{2}\right] \\
& y\left(E_{0}^{3}-E_{0}^{1}\right) \leq b_{3}-b_{1} \quad \forall y \in\left[\hat{y}_{1}, \hat{y}_{2}\right] \\
& y\left(E_{0}^{2}-E_{0}^{1}\right) \geq b_{2}-b_{1} \quad \forall y \in\left[\hat{y}_{2}, \hat{y}_{3}\right] \\
& y\left(E_{0}^{2}-E_{0}^{0}\right) \geq b_{2} \quad \forall y \in\left[\hat{y}_{2}, \hat{y}_{3}\right] \\
& y\left(E_{0}^{3}-E_{0}^{2}\right) \leq b_{3}-b_{2} \quad \forall y \in\left[\hat{y}_{2}, \hat{y}_{3}\right] \\
& y\left(E_{0}^{3}-E_{0}^{0}\right) \geq b_{3} \quad \forall y \geq \hat{y}_{3} \\
& y\left(E_{0}^{3}-E_{0}^{2}\right) \geq b_{3}-b_{2} \quad \forall y \geq \hat{y}_{3} \\
& y\left(E_{0}^{3}-E_{0}^{1}\right) \geq b_{3}-b_{1} \quad \forall y \geq \hat{y}_{3}
\end{aligned}
$$




$$
\begin{aligned}
& y\left(E_{1}^{1}-E_{1}^{0}\right) \leq b_{1} \quad \forall y \leq \hat{y}_{1} \\
& y\left(E_{1}^{2}-E_{1}^{0}\right) \leq b_{2} \quad \forall y \leq \hat{y}_{1} \\
& y\left(E_{1}^{3}-E_{1}^{0}\right) \leq b_{3} \quad \forall y \leq \hat{y}_{1} \\
& y\left(E_{1}^{2}-E_{1}^{1}\right) \geq b_{2}-b_{1} \forall y \in\left[\hat{y}_{2}, \hat{y}_{3}\right] \\
& y\left(E_{1}^{2}-E_{1}^{0}\right) \geq b_{2} \forall y \in\left[\hat{y}_{2}, \hat{y}_{3}\right] \\
& y\left(E_{1}^{3}-E_{1}^{2}\right) \leq b_{3}-b_{2} \forall y \in\left[\hat{y}_{2}, \hat{y}_{3}\right] \\
& y\left(E_{1}^{3}-E_{1}^{0}\right) \geq b_{3} \quad \forall y \geq \hat{y}_{3} \\
& y\left(E_{1}^{3}-E_{1}^{2}\right) \geq b_{3}-b_{2} \forall y \geq \hat{y}_{3} \\
& y\left(E_{1}^{3}-E_{1}^{1}\right) \geq b_{3}-b_{1} \quad \forall y \geq \hat{y}_{3}
\end{aligned}
$$

$$
\begin{aligned}
& y\left(E_{2}^{1}-E_{2}^{0}\right) \leq b_{1} \quad \forall y \leq \hat{y}_{1} \\
& y\left(E_{2}^{2}-E_{2}^{0}\right) \leq b_{2} \quad \forall y \leq \hat{y}_{1} \\
& y\left(E_{2}^{3}-E_{2}^{1}\right) \leq b_{3} \quad \forall y \leq \hat{y}_{1} \\
& y\left(E_{2}^{1}-E_{2}^{0}\right) \geq b_{1} \quad \forall y \in\left[\hat{y}_{1}, \hat{y}_{2}\right] \\
& y\left(E_{2}^{2}-E_{2}^{1}\right) \leq b_{2}-b_{1} \quad \forall y \in\left[\hat{y}_{1}, \hat{y}_{2}\right] \\
& y\left(E_{2}^{3}-E_{2}^{1}\right) \leq b_{3}-b_{1} \quad \forall y \in\left[\hat{y}_{1}, \hat{y}_{2}\right] \\
& y\left(E_{2}^{3}-E_{2}^{0}\right) \geq b_{3} \quad \forall y \geq \hat{y}_{3} \\
& y\left(E_{2}^{3}-E_{2}^{2}\right) \geq b_{3}-b_{2} \quad \forall y \geq \hat{y}_{3} \\
& y\left(E_{2}^{3}-E_{2}^{1}\right) \geq b_{3}-b_{1} \quad \forall y \geq \hat{y}_{3}
\end{aligned}
$$




$$
\begin{aligned}
& y\left(E_{3}^{1}-E_{3}^{0}\right) \leq b_{1} \quad \forall y \leq \hat{y}_{1} \\
& y\left(E_{3}^{2}-E_{3}^{0}\right) \leq b_{2} \quad \forall y \leq \hat{y}_{1} \\
& y\left(E_{3}^{3}-E_{3}^{0}\right) \leq b_{3} \quad \forall y \leq \hat{y}_{1} \\
& y\left(E_{3}^{1}-E_{3}^{0}\right) \geq b_{1} \quad \forall y \in\left[\hat{y}_{1}, \hat{y}_{2}\right] \\
& y\left(E_{3}^{2}-E_{3}^{0}\right) \leq b_{2}-b_{1} \quad \forall y \in\left[\hat{y}_{1}, \hat{y}_{2}\right] \\
& y\left(E_{3}^{3}-E_{3}^{0}\right) \leq b_{3}-b_{1} \quad \forall y \in\left[\hat{y}_{1}, \hat{y}_{2}\right] \\
& y\left(E_{3}^{2}-E_{3}^{1}\right) \geq b_{2}-b_{1} \forall y \in\left[\hat{y}_{2}, \hat{y}_{3}\right] \\
& y\left(E_{3}^{2}-E_{3}^{0}\right) \geq b_{2} \forall y \in\left[\hat{y}_{2}, \hat{y}_{3}\right] \\
& y\left(E_{3}^{3}-E_{3}^{2}\right) \leq b_{3}-b_{2} \quad \forall y \in\left[\hat{y}_{2}, \hat{y}_{3}\right]
\end{aligned}
$$

These conditions can be combined to give

$$
\begin{aligned}
& E_{0}^{1}-E_{0}^{0}=E_{1}^{1}-E_{1}^{0}=E_{2}^{1}-E_{2}^{0}=E_{3}^{1}-E_{3}^{0} \\
& E_{0}^{2}-E_{0}^{1}=E_{1}^{2}-E_{1}^{1}=E_{2}^{2}-E_{2}^{1}=E_{3}^{2}-E_{3}^{1} \\
& E_{0}^{3}-E_{0}^{2}=E_{1}^{3}-E_{1}^{2}=E_{2}^{3}-E_{2}^{2}=E_{3}^{3}-E_{3}^{2}
\end{aligned}
$$

For simplicity of notation, let me denote

$x=1-F\left(\hat{y}_{1}\right), \quad m=1-F\left(\hat{y}_{2}\right)+F\left(\hat{y}_{1}\right), \quad z=1-F\left(\hat{y}_{3}\right)+F\left(\hat{y}_{2}\right), \quad w=F\left(\hat{y}_{3}\right)$

Then (49) implies that

$$
\begin{gathered}
E_{2}^{3}-E_{2}^{2}=E_{0}^{3}-E_{0}^{2} \Longleftrightarrow \\
\beta z \hat{y}_{3}+(1-\beta z) E^{3}-E^{2}=\beta x \hat{y}_{3}+(1-\beta x) E^{3}-\beta x \hat{y}_{2}-(1-\beta x) E^{2} \\
\Longleftrightarrow \hat{y}_{2}=E^{2}+\frac{(z-x)}{x}\left(E^{3}-\hat{y}_{3}\right)
\end{gathered}
$$

(49) also implies that

$$
\begin{gathered}
E_{2}^{3}-E_{2}^{2}=E_{1}^{3}-E_{1}^{2} \Longleftrightarrow \\
\beta z \hat{y}_{3}+(1-\beta z) E^{3}-E^{2}=\beta m \hat{y}_{3}+(1-\beta m) E^{3}-\beta m \hat{y}_{2}-(1-\beta m) E^{2}
\end{gathered}
$$




$$
\Longleftrightarrow \hat{y}_{2}=E^{2}+\frac{(z-m)}{m}\left(E^{3}-\hat{y}_{3}\right)
$$

There are 2 conclusions that follow from (50) and (51): First, it has to be the case that $z<x$ and $z<m$ (implying that group 3 needs to be larger than both group 1 and group 2). Second, combining the two equations we find that $m=x$.

(48) implies that

$$
\begin{gathered}
E_{0}^{2}-E_{0}^{1}=E_{1}^{2}-E_{1}^{1} \Longleftrightarrow \\
\beta m \hat{y}_{2}+(1-\beta m) E^{2}-E^{1}=\beta x \hat{y}_{2}+(1-\beta x) E^{2}-\beta x \hat{y}_{1}-(1-\beta x) E^{1} \\
\Longleftrightarrow \hat{y}_{1}=\left(\frac{m-x}{x}\right)\left(E^{2}-\hat{y}_{2}\right)+E^{1}
\end{gathered}
$$

which implies that $m<x$ because $\hat{y}_{1}<E^{1}$. Hence, (48) and (49) cannot be satisfied at the same time, and a biased sorting equilibrium with consistency cannot exist for four groups. In fact, this argument holds also for more than four groups, because nowhere in this proof did I make the assumption that there are only four groups. The conditions that contradict each other would be the same with $n \geq 4$ groups. The analogous analysis can be conducted for proportional biased beliefs of type 2 to yield the exact same result: no biased sorting equilibria with global consistency will exist for more than three groups.

\subsubsection{The possibility of 3 groups}

Suppose there are 3 groups: A poor group with income from 0 to $\hat{y}_{1}$, a middle group with incomes from $\hat{y}_{1}$ to $\hat{y}_{2}$ and a rich group with incomes from $\hat{y}_{2}$ to $y_{\max }$. Suppose that the belief function is of the same type as in 
the previous section, and hence

$$
\begin{aligned}
& E_{0}^{0}=E^{0} \\
& E_{0}^{1}=\beta\left(1-F\left(\hat{y}_{1}\right)\right) \hat{y}_{1}+\left(1-\beta\left(1-F\left(\hat{y}_{1}\right)\right)\right) E^{1} \\
& E_{0}^{2}=\beta\left(1-F\left(\hat{y}_{1}\right)\right) \hat{y}_{2}+\left(1-\beta\left(1-F\left(\hat{y}_{1}\right)\right)\right) E^{2} \\
& E_{1}^{0}=\beta\left(1-F\left(\hat{y}_{2}\right)+F\left(\hat{y}_{1}\right)\right) \hat{y}_{1}+\left(1-\beta\left(1-F\left(\hat{y}_{2}\right)+F\left(\hat{y}_{1}\right)\right)\right) E^{0} \\
& E_{1}^{1}=E^{1} \\
& E_{1}^{2}=\beta\left(1-F\left(\hat{y}_{2}\right)+F\left(\hat{y}_{1}\right)\right) \hat{y}_{2}+\left(1-\beta\left(1-F\left(\hat{y}_{2}\right)+F\left(\hat{y}_{1}\right)\right)\right) E^{2} \\
& E_{2}^{0}=\beta\left(1-F\left(\hat{y}_{3}\right)+F\left(\hat{y}_{2}\right)\right) \hat{y}_{1}+\left(1-\beta\left(1-F\left(\hat{y}_{3}\right)+F\left(\hat{y}_{2}\right)\right)\right) E^{0} \\
& E_{2}^{1}=\beta\left(1-F\left(\hat{y}_{3}\right)+F\left(\hat{y}_{2}\right)\right) \hat{y}_{2}+\left(1-\beta\left(1-F\left(\hat{y}_{3}\right)+F\left(\hat{y}_{2}\right)\right)\right) E^{1} \\
& E_{2}^{2}=E^{2}
\end{aligned}
$$

Then in order for this partition to constitute a biased sorting equilibrium with global consistency, the following conditions have to hold:

For a biased sorting equilibrium, I need:

$$
\begin{aligned}
& y\left(E_{0}^{1}-E_{0}^{0}\right) \leq b_{1} \quad \forall y \leq \hat{y}_{1} \\
& y\left(E_{0}^{2}-E_{0}^{0}\right) \leq b_{2} \quad \forall y \leq \hat{y}_{1} \\
& y\left(E_{1}^{1}-E_{1}^{0}\right) \geq b_{1} \quad \forall y \in\left[\hat{y}_{1}, \hat{y}_{2}\right] \\
& y\left(E_{1}^{2}-E_{1}^{1}\right) \leq b_{2}-b_{1} \quad \forall y \in\left[\hat{y}_{1}, \hat{y}_{2}\right] \\
& y\left(E_{2}^{2}-E_{2}^{0}\right) \geq b_{2} \quad \forall y \geq \hat{y}_{2} \\
& y\left(E_{2}^{2}-E_{2}^{1}\right) \geq b_{2}-b_{1} \quad \forall y \geq \hat{y}_{2}
\end{aligned}
$$

For global consistency, the additional conditions are: 


$$
\begin{aligned}
y\left(E_{0}^{1}-E_{0}^{0}\right) & \geq b_{1} \quad \forall y \in\left[\hat{y}_{1}, \hat{y}_{2}\right] \\
y\left(E_{0}^{2}-E_{0}^{1}\right) & \leq b_{2}-b_{1} \quad \forall y \in\left[\hat{y}_{1}, \hat{y}_{2}\right] \\
y\left(E_{0}^{2}-E_{0}^{1}\right) & \geq b_{2}-b_{1} \quad \forall y \geq \hat{y}_{2} \\
y\left(E_{0}^{2}-E_{0}^{0}\right) & \geq b_{2} \quad \forall y \geq \hat{y}_{2} \\
j k y\left(E_{1}^{1}-E_{1}^{0}\right) & \leq b_{1} \quad \forall y \leq \hat{y}_{1} \\
y\left(E_{1}^{2}-E_{1}^{0}\right) & \leq b_{2} \quad \forall y \leq \hat{y}_{1} \\
y\left(E_{1}^{2}-E_{1}^{1}\right) & \geq b_{2}-b_{1} \quad \forall y \geq \hat{y}_{2} \\
y\left(E_{1}^{2}-E_{1}^{0}\right) & \geq b_{2} \quad \forall y \geq \hat{y}_{2} \\
y\left(E_{2}^{1}-E_{2}^{0}\right) & \leq b_{1} \quad \forall y \leq \hat{y}_{1} \\
y\left(E_{2}^{2}-E_{2}^{0}\right) & \leq b_{2} \quad \forall y \leq \hat{y}_{1} \\
y\left(E_{2}^{2}-E_{2}^{1}\right) & \leq b_{2}-b_{1} \quad \forall y \in\left[\hat{y}_{1}, \hat{y}_{2}\right] \\
y\left(E_{2}^{1}-E_{2}^{0}\right) & \geq b_{1} \quad \forall y \in\left[\hat{y}_{1}, \hat{y}_{2}\right]
\end{aligned}
$$

These conditions can be combined to

$$
\begin{aligned}
& E_{0}^{1}-E_{0}^{0}=E_{1}^{1}-E_{1}^{0}=E_{2}^{1}-E_{2}^{0} \\
& E_{0}^{2}-E_{0}^{1}=E_{1}^{2}-E_{1}^{1}=E_{2}^{2}-E_{2}^{1}
\end{aligned}
$$

Hence, $\hat{y}_{1}$ and $\hat{y}_{2}$ must be such that (52) and (53) are satisfied.

For simplicity of notation, let me denote

$$
x=1-F\left(\hat{y}_{1}\right), \quad m=1-F\left(\hat{y}_{2}\right)+F\left(\hat{y}_{1}\right), \quad z=F\left(\hat{y}_{2}\right)
$$

Then (52) implies

$$
\begin{gathered}
E_{1}^{1}-E_{1}^{0}=E_{2}^{1}-E_{2}^{0} \Longleftrightarrow \\
E^{1}-\beta m \hat{y}_{1}-(1-\beta m) E^{0}=\beta z \hat{y}_{2}+(1-\beta z) E^{1}-\beta z \hat{y}_{1}-(1-\beta z) E^{0} \\
\Longleftrightarrow z E^{1}+(z-m)\left(\hat{y}_{1}-E^{0}\right)=z \hat{y}_{2}
\end{gathered}
$$

which implies that $z>m$ because $\hat{y}_{2}>E^{1}$. 
Furthermore, from (53) I get

$$
\begin{gathered}
E_{0}^{2}-E_{0}^{1}=E_{1}^{2}-E_{1}^{1} \Longleftrightarrow \\
\beta x \hat{y}_{2}+(1-\beta x) E^{2}-\beta x \hat{y}_{1}-(1-\beta x) E^{1}=\beta m \hat{y}_{2}+(1-\beta m) E^{2}-E^{1} \\
\Longleftrightarrow \frac{m-x}{x}\left(E^{2}-\hat{y}_{2}\right)+E^{1}=\hat{y}_{1}
\end{gathered}
$$

and this implies $m<x$ because $\hat{y}_{1}<E^{1}$.

Moreover,

$$
E_{0}^{1}-E_{0}^{0}=E_{1}^{1}-E_{1}^{0}
$$

gives

$$
\hat{y}_{1}=\frac{x E^{1}+m E^{0}}{x+m} \text {. }
$$

Also,

$$
E_{1}^{2}-E_{1}^{1}=E_{2}^{2}-E_{2}^{1}
$$

gives

$$
\hat{y}_{2}=\frac{z E^{1}+m E^{2}}{z+m} .
$$

All together, these are 4 equations in 2 unknowns.

$$
\begin{gathered}
z E^{1}+(z-m)\left(\hat{y}_{1}-E^{0}\right)=z \hat{y}_{2} \\
\frac{m-x}{x}\left(E^{2}-\hat{y}_{2}\right)+E^{1}=\hat{y}_{1} \\
\hat{y}_{1}=\frac{x E^{1}+m E^{0}}{x+m} \\
\hat{y}_{2}=\frac{z E^{1}+m E^{2}}{z+m}
\end{gathered}
$$

Will such an equilibrium exist and be unique? Note that (56) and (57) are the same conditions as the equilibrium conditions (39) and (40) for the equilibrium with local consistency above. As I have argued, a pair $\left(\hat{y}_{1}^{*}, \hat{y}_{2}^{*}\right)$ satisfying these two conditions can exist (e.g. if the income distribution is uniform). It depends on $F($.$) whether a pair \left(\hat{y}_{1}^{*}, \hat{y}_{2}^{*}\right)$ satisfying all four of these equations exists. For instance, I have demonstrated above that such a pair $\left(\hat{y}_{1}^{*}, \hat{y}_{2}^{*}\right)$ cannot be found for the uniform distribution.

To conclude, the analysis in this section shows that biased sorting equi- 
libria with local consistency can, depending on the shape of the income distribution, exist for every (finite) number of groups. Whether biased sorting equilibria with global consistency with more than two groups can exist depends crucially on how misperceptions about non-neighbouring groups are specified. If they take the form of proportional biased beliefs, then equilibria with more than three groups can be ruled out. If the perceived difference in average incomes between groups is decreasing in group distance, equilibria with more than two groups can be ruled out. 